\title{
Lepton mixing and the charged-lepton mass ratios
}

\author{
Darius Jurčiukonis ${ }^{a}$ and Luís Lavoura ${ }^{b}$ \\ ${ }^{a}$ Institute of Theoretical Physics and Astronomy, University of Vilnius, \\ Sauletekio ave. 3, LT-10222 Vilnius, Lithuania \\ ${ }^{b}$ Instituto Superior Técnico, CFTP, Universidade de Lisboa, \\ 1049-001 Lisboa, Portugal \\ E-mail: darius.jurciukonis@tfai.vu.lt, balio@cftp.tecnico.ulisboa.pt
}

ABStRaCT: We construct a class of renormalizable models for lepton mixing that generate predictions given in terms of the charged-lepton mass ratios. We show that one of those models leads, when one takes into account the known experimental values, to almost maximal $C P$-breaking phases and to almost maximal neutrinoless double-beta decay. We study in detail the scalar potential of the models, especially the bounds imposed by unitarity on the values of the quartic couplings.

Keywords: Phenomenological Models

ArXiv EPrint: 1712.04292 


\section{Contents}

1 Introduction and notation 1

2 Models 3

2.1 Construction of the models 3

2.2 Classification of the models 6

$\begin{array}{lll}3 & \text { A specific model } & 7\end{array}$

4 The scalar potential $\quad 10$

4.1 Assumptions 10

4.2 The vacuum 11

$\begin{array}{ll}4.3 & \text { The scalar mass matrices } \\ \end{array}$

$\begin{array}{lll}4.4 & \text { The oblique parameter } T & 14\end{array}$

$\begin{array}{lll}\text { 4.5 Extension to the quark sector } & 15\end{array}$

$\begin{array}{lll}4.6 & \text { Procedure for producing the scatter plots } & 15\end{array}$

$\begin{array}{lll}4.7 & \text { Scatter plots } & 18\end{array}$

5 Conclusions $\quad 21$

A Unitarity bounds for a 3HDM with $\mathbb{Z}_{2} \times \mathbb{Z}_{2} \times \mathbb{Z}_{2}$ symmetry 23

A.1 General case 23

A.2 Case with additional symmetry $\phi_{2} \leftrightarrow \phi_{3} \quad 26$

$\begin{array}{ll}\text { B Other stability points } & 26\end{array}$

\section{Introduction and notation}

In this paper we present a class of renormalizable models that are extensions of the Standard Model $(\mathrm{SM})$. The models have gauge group $\mathrm{SU}(2) \times \mathrm{U}(1)$ just as the SM. They feature an extended scalar sector, with three $\mathrm{SU}(2)$ doublets $\phi_{k}=\left(\phi_{k}^{+}, \phi_{k}^{0}\right)^{T}(k=1,2,3)$ instead of one; we let $\tilde{\phi}_{k}=\left(\phi_{k}^{0^{*}},-\phi_{k}^{-}\right)^{T}$ denote the conjugate doublets. The leptonic sector is also extended, with the addition to the SM of three right-handed (i.e. SU(2)-singlet) neutrinos $\nu_{R \psi},{ }^{1}$ which enable a type-I seesaw mechanism [1-5] to suppress the standard-neutrino masses. Our models have family-lepton-number symmetries

$$
D_{L \psi} \rightarrow e^{i \xi_{\psi}} D_{L \psi}, \quad \psi_{R} \rightarrow e^{i \xi_{\psi}} \psi_{R}, \quad \nu_{R \psi} \rightarrow e^{i \xi_{\psi}} \nu_{R \psi},
$$

\footnotetext{
${ }^{1}$ In this paper the Greek letters $\psi, \alpha, \beta$, and $\gamma$ in general run over the lepton flavours $e, \mu$, and $\tau$. Whenever we use $(\alpha, \beta, \gamma)$ we mean a permutation of $(e, \mu, \tau)$, i.e. $\alpha \neq \beta \neq \gamma \neq \alpha$.
} 
where the phases $\xi_{e}, \xi_{\mu}$, and $\xi_{\tau}$ are arbitrary and uncorrelated. In transformation (1.1), $\psi_{R}$ denotes the right-handed charged leptons and $D_{L \psi}=\left(\nu_{L \psi}, \psi_{L}\right)^{T}$ denotes the $\mathrm{SU}(2)$ doublets of left-handed neutrinos $\nu_{L \psi}$ and charged leptons $\psi_{L}$. In our models both the charged-lepton mass matrix $M_{\ell}$ and the neutrino Dirac mass matrix $M_{D}$ are diagonal, because they originate in Yukawa couplings that respect the family-lepton-number symmetries (1.1). Thus,

$$
M_{\ell}=\operatorname{diag}\left(\ell_{e}, \ell_{\mu}, \ell_{\tau}\right), \quad M_{D}=\operatorname{diag}\left(D_{e}, D_{\mu}, D_{\tau}\right)
$$

where

$$
\left|\ell_{\psi}\right|=m_{\psi}
$$

are the charged-lepton masses. The symmetries (1.1) leave the Yukawa couplings invariant but they are broken softly by the Majorana mass terms of the right-handed neutrinos, given by

$$
\mathcal{L}_{\mathrm{Maj}}=-\frac{1}{2}\left(\overline{\nu_{e R}}, \overline{\nu_{\mu R}}, \overline{\nu_{\tau R}}\right) M_{R} C\left(\begin{array}{c}
\overline{\bar{\nu}_{e R}} T \\
\overline{\bar{\nu}_{\mu R}} T \\
\overline{\nu_{\tau R}} T
\end{array}\right)+\text { H.c. }
$$

In equation (1.4), $C$ is the charge-conjugation matrix in Dirac space; the $3 \times 3$ flavour-space matrix $M_{R}$ is symmetric. The seesaw mechanism produces an effective light-neutrino mass matrix $M=-M_{D} M_{R}^{-1} M_{D}$, i.e.

$$
M_{\psi \psi^{\prime}}=-D_{\psi} D_{\psi^{\prime}}\left(M_{R}^{-1}\right)_{\psi \psi^{\prime}}, \quad \forall \psi, \psi^{\prime} \in\{e, \mu, \tau\} .
$$

Note that, since $M_{D}$ and $M_{\ell}$ are diagonal, the matrix $M_{R}$ is the sole origin of lepton mixing in our models $[6,7] .^{2}$ The symmetric matrix $M$ is diagonalized as

$$
U^{T} M U=\operatorname{diag}\left(m_{1}, m_{2}, m_{3}\right),
$$

where the $m_{k}$ are the (non-negative real) light-neutrino masses and $U$ is the lepton mixing matrix, for which we use the standard parameterization [9]

$$
\begin{aligned}
U= & \left(\begin{array}{ccc}
c_{12} c_{13} & s_{12} c_{13} & s_{13} e^{-i \delta} \\
-s_{12} c_{23}-c_{12} s_{23} s_{13} e^{i \delta} & c_{12} c_{23}-s_{12} s_{23} s_{13} e^{i \delta} & s_{23} c_{13} \\
s_{12} s_{23}-c_{12} c_{23} s_{13} e^{i \delta} & -c_{12} s_{23}-s_{12} c_{23} s_{13} e^{i \delta} & c_{23} c_{13}
\end{array}\right) \\
& \times \operatorname{diag}\left(1, e^{i \alpha_{21} / 2}, e^{i \alpha_{31} / 2}\right)
\end{aligned}
$$

where $c_{i j}=\cos \theta_{i j}$ and $s_{i j}=\sin \theta_{i j}$ for $i j=12,23,13$. The phase $\delta$ is the Dirac phase; $\alpha_{21}$ and $\alpha_{31}$ are the Majorana phases.

\footnotetext{
${ }^{2}$ In the study of leptogenesis one uses a basis for the mass matrices where $M_{\ell}$ and $M_{R}$ are diagonal but $M_{D}$ is not. In our models $M_{\ell}$ and $M_{D}$ are diagonal but $M_{R}$ is not. If in our models $M_{R}$ is diagonalized as $V^{T} M_{R} V=\operatorname{diag}\left(M_{1}, M_{2}, M_{3}\right)$, where $V$ is a $3 \times 3$ unitary matrix and $M_{1,2,3}$ are non-negative real, then $M_{D}^{\prime}=V^{T} M_{D}$ in the basis appropriate for the computation of leptogenesis. The Hermitian matrix relevant for leptogenesis is $R \equiv M_{D}^{\prime} M_{D}^{\prime \dagger}=V^{T} M_{D} M_{D}^{\dagger} V^{*}$, which is non-diagonal. Thus, leptogenesis is in principle viable in our models. See ref. [8] for details.
} 
The purpose of our models is to make predictions for the matrix $U$. There are in the literature many predictive models for $U ;^{3}$ the models in this paper are original in that they are well-defined renormalizable models that produce predictions for the neutrino mass matrix $M$ in terms of charged-lepton mass ratios. Since the mass ratios $m_{\mu} / m_{\tau}, m_{e} / m_{\mu}$, and $m_{e} / m_{\tau}$ are very small, the predictions of our models are hardly distinguishable in practice from the cases with 'texture zeroes' in the neutrino mass matrix [13].

In section 2 we expound the construction of the models and classify the various models that our class of models encompasses. Section 3 focusses on a specific model with remarkable predictions: almost-maximal $\delta$ and almost-maximal neutrinoless double-beta decay. Section 4 discusses a scalar potential for our models and the way in which that potential is able to reproduce the Higgs particle discovered at the LHC. Section 5 contains our main conclusions. Appendix A deals on the derivation of the unitarity bounds on the coupling constants of the scalar potential. In appendix B we compute the expectation value of the scalar potential in the various stability points of that potential.

\section{Models}

\subsection{Construction of the models}

Our class of models may be divided into four subclasses:

1. Models with Yukawa Lagrangian

$$
\begin{aligned}
\mathcal{L}_{1}= & -y_{1} \overline{D_{L \alpha}} \nu_{R \alpha} \tilde{\phi}_{1}-y_{2} \overline{D_{L \alpha}} \alpha_{R} \phi_{1} \\
& -y_{3}\left(\overline{D_{L \beta}} \nu_{R \beta} \tilde{\phi}_{2}+\overline{D_{L \gamma}} \nu_{R \gamma} \tilde{\phi}_{3}\right) \\
& -y_{4}\left(\overline{D_{L \beta}} \beta_{R} \phi_{2}+\overline{D_{L \gamma}} \gamma_{R} \phi_{3}\right)+\text { H.c. },
\end{aligned}
$$

where $(\alpha, \beta, \gamma)$ is a permutation of $(e, \mu, \tau)$ and $y_{1,2,3,4}$ are Yukawa coupling constants, which are in general complex.

2. Models with Yukawa Lagrangian

$$
\begin{aligned}
\mathcal{L}_{2}= & -y_{1} \overline{D_{L \alpha}} \nu_{R \alpha} \tilde{\phi}_{1}-y_{2} \overline{D_{L \alpha}} \alpha_{R} \phi_{1} \\
& -y_{3}\left(\overline{D_{L \beta}} \nu_{R \beta} \tilde{\phi}_{2}+\overline{D_{L \gamma}} \nu_{R \gamma} \tilde{\phi}_{3}\right) \\
& -y_{4}\left(\overline{D_{L \beta}} \beta_{R} \phi_{3}+\overline{D_{L \gamma}} \gamma_{R} \phi_{2}\right)+\text { H.c. }
\end{aligned}
$$

Note that $\mathcal{L}_{1}$ and $\mathcal{L}_{2}$ differ only in their last lines.

3. Models with Yukawa Lagrangian

$$
\begin{aligned}
\mathcal{L}_{3}= & -y_{1} \overline{D_{L \alpha}} \nu_{R \alpha} \tilde{\phi}_{1}-y_{2} \overline{D_{L \alpha}} \alpha_{R} \phi_{1} \\
& -y_{3} \overline{D_{L \beta}} \nu_{R \beta} \tilde{\phi}_{2}-y_{3}^{*} \overline{D_{L \gamma}} \nu_{R \gamma} \tilde{\phi}_{3} \\
& -y_{4} \overline{D_{L \beta}} \beta_{R} \phi_{2}-y_{4}^{*} \overline{D_{L \gamma}} \gamma_{R} \phi_{3}+\text { H.c. },
\end{aligned}
$$

where $y_{1}$ and $y_{2}$ are real while $y_{3}$ and $y_{4}$ are in general complex.

\footnotetext{
${ }^{3}$ See the reviews [10-12]; the original papers are in the bibliographies of those reviews.
} 
4. Models with Yukawa Lagrangian

$$
\begin{aligned}
\mathcal{L}_{4}= & -y_{1} \overline{D_{L \alpha}} \nu_{R \alpha} \tilde{\phi}_{1}-y_{2} \overline{D_{L \alpha}} \alpha_{R} \phi_{1} \\
& -y_{3} \overline{D_{L \beta}} \nu_{R \beta} \tilde{\phi}_{2}-y_{3}^{*} \overline{D_{L \gamma}} \nu_{R \gamma} \tilde{\phi}_{3} \\
& -y_{4} \overline{D_{L \beta}} \beta_{R} \phi_{3}-y_{4}^{*} \overline{D_{L \gamma}} \gamma_{R} \phi_{2}+\text { H.c. },
\end{aligned}
$$

where once again $y_{1}$ and $y_{2}$ are real. The Lagrangians (2.3) and (2.4) differ in their last lines.

It is clear that $\mathcal{L}_{1,2,3,4}$ enjoy the family-lepton-number symmetries (1.1). The Lagrangians (2.1) and (2.2) further enjoy the interchange symmetry

$$
\phi_{2} \leftrightarrow \phi_{3}, \quad D_{L \beta} \leftrightarrow D_{L \gamma}, \quad \beta_{R} \leftrightarrow \gamma_{R}, \quad \nu_{R \beta} \leftrightarrow \nu_{R \gamma} .
$$

The Lagrangians (2.3) and (2.4) are invariant under the $C P$ symmetry

$$
\begin{aligned}
\phi_{1}(x) & \rightarrow \phi_{1}^{*}(\bar{x}), & \phi_{2}(x) & \rightarrow \phi_{3}^{*}(\bar{x}), & \phi_{3}(x) & \rightarrow \phi_{2}^{*}(\bar{x}), \\
\alpha_{R}(x) & \rightarrow K{\overline{\alpha_{R}}}^{T}(\bar{x}), & \beta_{R}(x) & \rightarrow K{\overline{\gamma_{R}}}^{T}(\bar{x}), & \gamma_{R}(x) & \rightarrow K{\overline{\beta_{R}}}^{T}(\bar{x}), \\
\nu_{R \alpha}(x) & \rightarrow K{\overline{\nu_{R \alpha}}}^{T}(\bar{x}), & \nu_{R \beta}(x) & \rightarrow K{\overline{\nu_{R \gamma}}}^{T}(\bar{x}), & \nu_{R \gamma}(x) & \rightarrow K{\overline{\nu_{R \beta}}}^{T}(\bar{x}), \\
D_{L \alpha}(x) & \rightarrow K{\overline{D_{L \alpha}}}^{T}(\bar{x}), & D_{L \beta}(x) & \rightarrow K{\overline{D_{L \gamma}}}^{T}(\bar{x}), & D_{L \gamma}(x) & \rightarrow K{\overline{D_{L \beta}}}^{T}(\bar{x}),
\end{aligned}
$$

where $x \equiv(t, \vec{r})$ and $\bar{x} \equiv(t,-\vec{r}) ; K \equiv i \gamma_{0} C$ is the $C P$-transformation matrix in Dirac space. Moreover, in the last line of transformation (2.6),

$$
{\overline{D_{L \psi}}}^{T} \equiv\left(\begin{array}{c}
{\overline{\nu_{L \psi}}}^{T} \\
{\overline{\psi_{L}}}^{T}
\end{array}\right) .
$$

The $C P$ transformation (2.6) interchanges the lepton flavours $\beta$ and $\gamma$.

The Lagrangians (2.1)-(2.4) necessitate additional symmetries to guarantee that each scalar doublet only couples to the desired lepton flavour. There is a large arbitrariness in the choice of the additional symmetries. In this paper we choose them to be

$$
\mathbb{Z}_{2}^{(1)}: \quad \phi_{1} \rightarrow-\phi_{1}, \quad D_{L \alpha} \rightarrow-D_{L \alpha},
$$

for all four Lagrangians (2.1)-(2.4); and either

$$
\begin{array}{lll}
\mathbb{Z}_{2}^{(2)}: & \phi_{2} \rightarrow-\phi_{2}, \quad \beta_{R} \rightarrow-\beta_{R}, \quad \nu_{R \beta} \rightarrow-\nu_{R \beta}, \\
\mathbb{Z}_{2}^{(3)}: & \phi_{3} \rightarrow-\phi_{3}, \quad \gamma_{R} \rightarrow-\gamma_{R}, \quad \nu_{R \gamma} \rightarrow-\nu_{R \gamma},
\end{array}
$$

for Lagrangians (2.1) and (2.3), or else

$$
\begin{array}{ll}
\mathbb{Z}_{2}^{(4)}: & \phi_{2} \rightarrow-\phi_{2}, \quad \gamma_{R} \rightarrow-\gamma_{R}, \quad \nu_{R \beta} \rightarrow-\nu_{R \beta}, \\
\mathbb{Z}_{2}^{(5)}: & \phi_{3} \rightarrow-\phi_{3}, \quad \beta_{R} \rightarrow-\beta_{R}, \quad \nu_{R \gamma} \rightarrow-\nu_{R \gamma},
\end{array}
$$

for Lagrangians (2.2) and (2.4). The transformations (2.8) and either (2.9) or (2.10) form a $\mathbb{Z}_{2} \times \mathbb{Z}_{2} \times \mathbb{Z}_{2}$ symmetry. 
Let $v_{k}$ denote the vacuum expectation value $(\mathrm{VEV})$ of $\phi_{k}^{0}$. Then, from $\mathcal{L}_{1}$,

$$
\begin{gathered}
\left(M_{\ell}\right)_{\alpha \alpha} \equiv \ell_{\alpha}=y_{2} v_{1}, \quad\left(M_{\ell}\right)_{\beta \beta} \equiv \ell_{\beta}=y_{4} v_{2}, \quad\left(M_{\ell}\right)_{\gamma \gamma} \equiv \ell_{\gamma}=y_{4} v_{3} \\
\left(M_{D}\right)_{\alpha \alpha} \equiv D_{\alpha}=y_{1}^{*} v_{1}, \quad\left(M_{D}\right)_{\beta \beta} \equiv D_{\beta}=y_{3}^{*} v_{2}, \quad\left(M_{D}\right)_{\gamma \gamma} \equiv D_{\gamma}=y_{3}^{*} v_{3}
\end{gathered}
$$

for model 1. From the Yukawa Lagrangian (2.2),

$$
\begin{aligned}
\ell_{\alpha} & =y_{2} v_{1}, \quad \ell_{\beta}=y_{4} v_{3}, \quad \ell_{\gamma}=y_{4} v_{2}, \\
D_{\alpha} & =y_{1}^{*} v_{1}, \quad D_{\beta}=y_{3}^{*} v_{2}, \quad D_{\gamma}=y_{3}^{*} v_{3}
\end{aligned}
$$

for model 2. From $\mathcal{L}_{3}$,

$$
\begin{aligned}
\ell_{\alpha} & =y_{2} v_{1}, \quad \ell_{\beta}=y_{4} v_{2}, \quad \ell_{\gamma}=y_{4}^{*} v_{3}, \\
D_{\alpha} & =y_{1}^{*} v_{1}, \quad D_{\beta}=y_{3}^{*} v_{2}, \quad D_{\gamma}=y_{3} v_{3}
\end{aligned}
$$

for model 3. From the Yukawa Lagrangian (2.4),

$$
\begin{aligned}
\ell_{\alpha} & =y_{2} v_{1}, \quad \ell_{\beta}=y_{4} v_{3}, \quad \ell_{\gamma}=y_{4}^{*} v_{2}, \\
D_{\alpha} & =y_{1}^{*} v_{1}, \quad D_{\beta}=y_{3}^{*} v_{2}, \quad D_{\gamma}=y_{3} v_{3}
\end{aligned}
$$

for model 4.

We next consider the right-handed-neutrino Majorana mass terms. They softly break the lepton-number symmetries (1.1) and also the additional symmetries (2.9) or (2.10). We assume that they do not break either the interchange symmetry (2.5) of models 1 and 2 or the $C P$ symmetry (2.6) of models 3 and 4. This means that, in models 1 and 2 ,

$$
\left(M_{R}\right)_{\beta \beta}=\left(M_{R}\right)_{\gamma \gamma}, \quad\left(M_{R}\right)_{\alpha \beta}=\left(M_{R}\right)_{\alpha \gamma} .
$$

Clearly, the symmetry (2.15) for the matrix $M_{R}$ is also valid for the matrix $M_{R}^{-1}$. Therefore, from equation (1.5),

$$
\frac{M_{\beta \beta}}{M_{\gamma \gamma}}=\left(\frac{D_{\beta}}{D_{\gamma}}\right)^{2}, \quad \frac{M_{\alpha \beta}}{M_{\alpha \gamma}}=\frac{D_{\beta}}{D_{\gamma}}
$$

for models 1 and 2. This means that the rephasing-invariant phase

$$
\arg \left[M_{\gamma \gamma}\left(M_{\alpha \beta}\right)^{2} M_{\beta \beta}^{*}\left(M_{\alpha \gamma}^{*}\right)^{2}\right]=0
$$

in models 1 and 2. Additionally, from equations (2.11) and (1.3),

$$
\left|\frac{M_{\beta \beta}}{M_{\gamma \gamma}}\right|=\frac{m_{\beta}^{2}}{m_{\gamma}^{2}}, \quad\left|\frac{M_{\alpha \beta}}{M_{\alpha \gamma}}\right|=\frac{m_{\beta}}{m_{\gamma}}
$$

for model 1; while, from equations (2.12) and (1.3),

$$
\left|\frac{M_{\beta \beta}}{M_{\gamma \gamma}}\right|=\frac{m_{\gamma}^{2}}{m_{\beta}^{2}}, \quad\left|\frac{M_{\alpha \beta}}{M_{\alpha \gamma}}\right|=\frac{m_{\gamma}}{m_{\beta}}
$$

for model 2. 
We conclude that model 1 makes three predictions for the effective light-neutrino mass matrix $M$ : equations (2.17) and (2.18). Model 2 also makes three predictions: equations (2.17) and (2.19).

In models 3 and 4 , we assume that the $C P$ symmetry (2.6) is not broken by the Majorana mass terms of the $\nu_{R}$. This means that

$$
\begin{array}{ll}
\left(M_{R}\right)_{\beta \beta}=\left(M_{R}^{*}\right)_{\gamma \gamma}, & \left(M_{R}\right)_{\alpha \beta}=\left(M_{R}^{*}\right)_{\alpha \gamma}, \\
\left(M_{R}\right)_{\alpha \alpha}=\left(M_{R}^{*}\right)_{\alpha \alpha}, & \left(M_{R}\right)_{\beta \gamma}=\left(M_{R}^{*}\right)_{\beta \gamma}
\end{array}
$$

in those models. Equations $(2.20)$ are valid for $M_{R}^{-1}$ as weall as for $M_{R}$, hence

$$
\frac{M_{\beta \beta}}{M_{\gamma \gamma}^{*}}=\frac{D_{\beta} D_{\beta}}{D_{\gamma}^{*} D_{\gamma}^{*}}, \quad \frac{M_{\alpha \beta}}{M_{\alpha \gamma}^{*}}=\frac{D_{\alpha} D_{\beta}}{D_{\alpha}^{*} D_{\gamma}^{*}} \quad \frac{M_{\alpha \alpha}}{M_{\alpha \alpha}^{*}}=\frac{D_{\alpha} D_{\alpha}}{D_{\alpha}^{*} D_{\alpha}^{*}}, \quad \frac{M_{\beta \gamma}}{M_{\beta \gamma}^{*}}=\frac{D_{\beta} D_{\gamma}}{D_{\beta}^{*} D_{\gamma}^{*}}
$$

for models 3 and 4. Equations (2.21) imply the following rephasing-invariant conditions on the matrix $M$ :

$$
\begin{aligned}
\arg \left[M_{\beta \beta}^{*} M_{\gamma \gamma}^{*}\left(M_{\beta \gamma}\right)^{2}\right] & =0, \\
\arg \left(M_{\alpha \alpha}^{*} M_{\beta \gamma}^{*} M_{\alpha \beta} M_{\alpha \gamma}\right) & =0 .
\end{aligned}
$$

Moreover, from equations (1.3) and (2.13) one derives equation (2.18), which is thus also valid for model 3; from equations (1.3) and (2.14) one derives equation (2.19), which thus applies to model 4 . We conclude that model 3 makes four predictions for $M$ : equations (2.22) and (2.18). Model 4 also makes four predictions: equations (2.22) and (2.19).

\subsection{Classification of the models}

Our class of models encompasses twelve models, depending on whether one uses model 1 , 2,3 , or 4 and depending on whether the flavour $\alpha$ is taken to be $e, \mu$, or $\tau$. (The flavours $\beta$ and $\gamma$ are treated symetrically in the models.)

There is a distinction between the models with interchange symmetry (2.5) and the models with $C P$ symmetry (2.6): the former lead to only one constraint (2.17) on the phases of the matrix elements of $M$, while the latter lead to the two constraints (2.22). The $C P$ symmetry (2.6) is more powerful than the interchange symmetry (2.5).

However, in practice the distinction between equation (2.17) and equations (2.22) is not very significant, because the charged-lepton mass ratios are so small that they force some $M$-matrix elements to be very close to zero, hence their phases do not matter much. We see from equations (2.18) and (2.19) that our twelve models may be classified in six types:

i. Models that predict

$$
\left|\frac{M_{e e}}{M_{\mu \mu}}\right|=\frac{m_{e}^{2}}{m_{\mu}^{2}}, \quad\left|\frac{M_{e \tau}}{M_{\mu \tau}}\right|=\frac{m_{e}}{m_{\mu}}
$$

Since $m_{e} \ll m_{\mu}$, in these models one is close to the situation $M_{e e}=M_{e \tau}=0$, which is case $\mathrm{A}_{2}$ of ref. [13]. 
ii. Models that predict

$$
\left|\frac{M_{e e}}{M_{\mu \mu}}\right|=\frac{m_{\mu}^{2}}{m_{e}^{2}}, \quad\left|\frac{M_{e \tau}}{M_{\mu \tau}}\right|=\frac{m_{\mu}}{m_{e}} .
$$

Since $m_{e} \ll m_{\mu}$ these models predict $M_{\mu \mu} \approx 0$ and $M_{\mu \tau} \approx 0$. According to ref. [13], $M_{\mu \mu}=M_{\mu \tau}=0$ is phenomenologically excluded.

iii. Models that predict

$$
\left|\frac{M_{e e}}{M_{\tau \tau}}\right|=\frac{m_{e}^{2}}{m_{\tau}^{2}}, \quad\left|\frac{M_{e \mu}}{M_{\mu \tau}}\right|=\frac{m_{e}}{m_{\tau}} .
$$

Since $m_{e} \ll m_{\tau}$ these models predict $M_{e e} \approx 0$ and $M_{e \mu} \approx 0$. They are therefore close to case $\mathrm{A}_{1}$ of ref. [13].

iv. Models that predict

$$
\left|\frac{M_{e e}}{M_{\tau \tau}}\right|=\frac{m_{\tau}^{2}}{m_{e}^{2}}, \quad\left|\frac{M_{e \mu}}{M_{\mu \tau}}\right|=\frac{m_{\tau}}{m_{e}} .
$$

This leads to, approximately, $M_{\tau \tau}=M_{\mu \tau}=0$, which is phenomenologically excluded.

v. Models that predict

$$
\left|\frac{M_{\mu \mu}}{M_{\tau \tau}}\right|=\frac{m_{\mu}^{2}}{m_{\tau}^{2}}, \quad\left|\frac{M_{e \mu}}{M_{e \tau}}\right|=\frac{m_{\mu}}{m_{\tau}} .
$$

Since $m_{\mu} \ll m_{\tau}$ these models predict $M_{\mu \mu} \approx 0$ and $M_{e \mu} \approx 0$. They are therefore close to case $\mathrm{B}_{3}$ of ref. [13].

vi. Models that predict

$$
\left|\frac{M_{\mu \mu}}{M_{\tau \tau}}\right|=\frac{m_{\tau}^{2}}{m_{\mu}^{2}}, \quad\left|\frac{M_{e \mu}}{M_{e \tau}}\right|=\frac{m_{\tau}}{m_{\mu}} .
$$

This leads to $M_{\tau \tau} \approx 0$ and $M_{e \tau} \approx 0$, corresponding to case $\mathrm{B}_{4}$ of ref. [13].

We thus find that, out of our twelve models, four should be phenomenologically excluded. The other eight are viable; two of them approximately coincide in their predictions with case $A_{1}$ of ref. [13], two other with case $A_{2}$, two more with case $B_{3}$, and the last two with case $\mathrm{B}_{4}$.

We have made numerical simulations of all our models and they very much vindicate the above conclusions. We do not feel it worth presenting those numerical simulations in detail here. In the next section we focus solely on one model that in our opinion yields particularly interesting results.

\section{A specific model}

In this section we deal on one of our models, which predicts

$$
\begin{aligned}
\left|\frac{M_{\mu \mu}}{M_{\tau \tau}}\right| & =\frac{m_{\mu}^{2}}{m_{\tau}^{2},} \\
\left|\frac{M_{e \mu}}{M_{e \tau}}\right| & =\frac{m_{\mu}}{m_{\tau}}, \\
\arg \left[M_{\tau \tau}\left(M_{e \mu}\right)^{2} M_{\mu \mu}^{*}\left(M_{e \tau}^{*}\right)^{2}\right] & =0 .
\end{aligned}
$$


Equations (3.1) are three predictions. This is not much; for instance, each of the cases with two texture zeroes of ref. [13] has four predictions, and there are models with as many as six predictions for $M$. So, one might think that the predictions (3.1) are of little practical consequence. This is not so, however.

We use $M=U^{*} \operatorname{diag}\left(m_{1}, m_{2}, m_{3}\right) U^{\dagger}$ and the parameterization of $U$ in equation (1.7). We also use the experimental $3 \sigma$ bounds [14]

$$
\begin{aligned}
7.05 & \leq \frac{m_{2}^{2}-m_{1}^{2}}{10^{-5} \mathrm{eV}^{2}} \leq 8.14, \\
0.273 & \leq s_{12}^{2} \leq 0.379 \\
0.0189 & \leq s_{13}^{2} \leq 0.0239,
\end{aligned}
$$

and either

$$
\begin{aligned}
& 2.43 \leq \frac{m_{3}^{2}-m_{1}^{2}}{10^{-3} \mathrm{eV}^{2}} \leq 2.67 \\
& 0.384 \leq s_{23}^{2} \leq 0.635
\end{aligned}
$$

for a normal ordering of the neutrino masses, or

$$
\begin{aligned}
& 2.37 \leq \frac{m_{1}^{2}-m_{3}^{2}}{10^{-3} \mathrm{eV}^{2}} \leq 2.61, \\
& 0.388 \leq s_{23}^{2} \leq 0.638
\end{aligned}
$$

for the inverted ordering of the neutrino masses. The phases $\delta, \alpha_{21}$, and $\alpha_{31}$ are unknown, just as the overall scale of the neutrino masses; we represent the latter through $m_{\text {sum }} \equiv m_{1}+m_{2}+m_{3}$. Strong cosmological arguments suggest that $m_{\text {sum }} \leq 0.25 \mathrm{eV}$ at $95 \%$ confidence level [15]. ${ }^{4}$

A quantity of especial importance is

$$
m_{\beta \beta} \equiv\left|M_{e e}\right|=\left|m_{1} c_{12}^{2} c_{13}^{2}+m_{2} s_{12}^{2} c_{13}^{2} e^{i \alpha_{21}}+m_{3} s_{13}^{2} e^{i\left(\alpha_{31}-2 \delta\right)}\right| .
$$

This quantity is relevant for neutrinoless double-beta decay, which should proceed with a rate approximately proportional to $m_{\beta \beta}^{2}$. It is clear that $m_{\beta \beta}$ becomes maximal when

$$
\alpha_{21}=0, \quad \alpha_{31}=2 \delta
$$

for whatever value of the phase $\delta$.

In figure 1 we have plotted $m_{\beta \beta}$ as a function of $m_{\text {sum }}$, both when only the inequalities (3.2) and either (3.3) or (3.4) hold, and when furthermore the predictions (3.1) are enforced. The information in that figure is clear: the predictions (3.1) lead to almost maximal $m_{\beta \beta}$, irrespective of the neutrino mass ordering.

This of course happens because equations (3.6) hold. In figure 2 one observes that this is indeed so and that, moreover, the predictions (3.1) lead to $\delta \approx 3 \pi / 2$. Thus, our model firmly predicts the three phases $\delta, \alpha_{21}$, and $\alpha_{31}$; the phase $\delta$ is predicted to be very close to $1.5 \pi$, and this agrees nicely with its $1 \sigma$-preferred experimental value [14].

\footnotetext{
${ }^{4} \mathrm{~A}$ recent paper [16] claims that $m_{\text {sum }}=0.11 \pm 0.03 \mathrm{eV}$.
} 

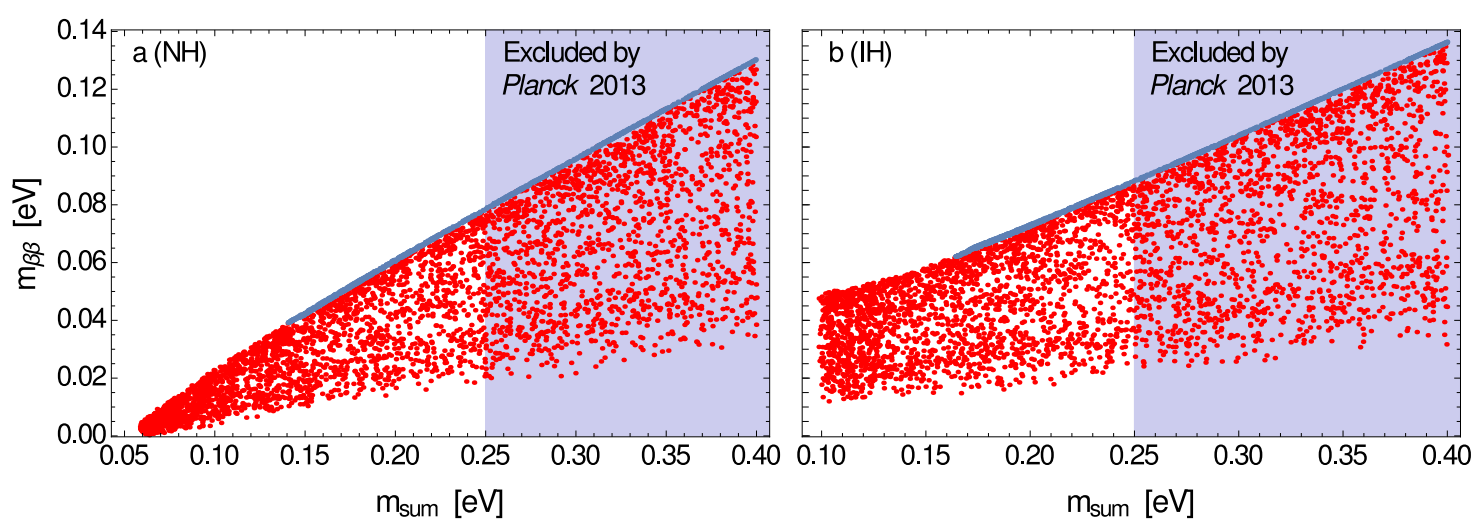

Figure 1. Scatter plots of $m_{\beta \beta}$ versus $m_{\text {sum. }}$. Figure 1a is for a normal ordering of the neutrino masses and figure $1 \mathrm{~b}$ is for an inverted ordering. The red points take into account only the experimental bounds (3.2)-(3.4); the blue points arise from the constraints (3.1).
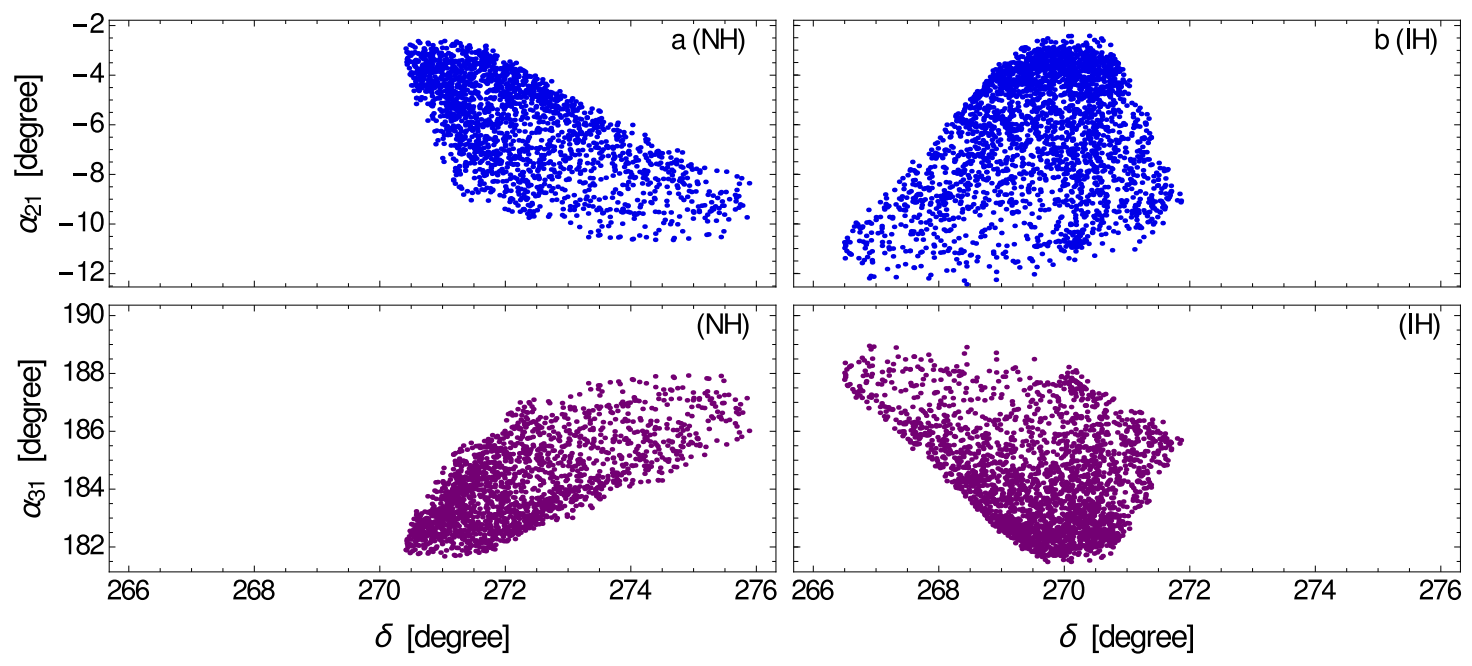

Figure 2. Scatter plots of the phases $\delta, \alpha_{21}$, and $\alpha_{31}$ following from the constraints (3.1). Figure 2a is for a normal ordering of the neutrino masses and figure $2 \mathrm{~b}$ is for an inverted ordering.

One moreover observes in figure 1 that our model does not tolerate very low neutrino masses, but goes well with almost-degenerate neutrinos: $m_{\text {sum }} \gtrsim 0.15 \mathrm{eV}$ for both the normal and inverted neutrino mass spectra.

This specific model does not just predict the Dirac and Majorana phases; it moreover predicts the quadrant of the angle $\theta_{23}$ and a correlation between that angle and $m_{\text {sum. }}$. That is observed in figure 3 . One sees that $\theta_{23}$ lies in the first quadrant when the neutrino mass ordering is normal, in the second quadrant when it is inverted. One also sees that $\theta_{23}$ is correlated with $m_{\text {sum }}$, with $\theta_{23}$ becoming ever closer to $\pi / 4$ when $m_{\text {sum }}$ grows.

Figures 1 and 3 are very similar to analogous figures displayed in ref. [17] for case $\mathrm{B}_{3}$ of ref. [13]. That case is defined by $M_{\mu \mu}=M_{e \mu}=0,{ }^{5}$ which of course means four pre-

\footnotetext{
${ }^{5}$ The paper of ref. [13] contains various two-texture-zero cases, in particular case $\mathrm{B}_{3}$ defined as $M_{\mu \mu}=M_{e \mu}=0$. The cases are of course not full models. However, it was demonstrated in ref. [18] that any texture-zero mass matrix may result from a renormalizable model.
} 

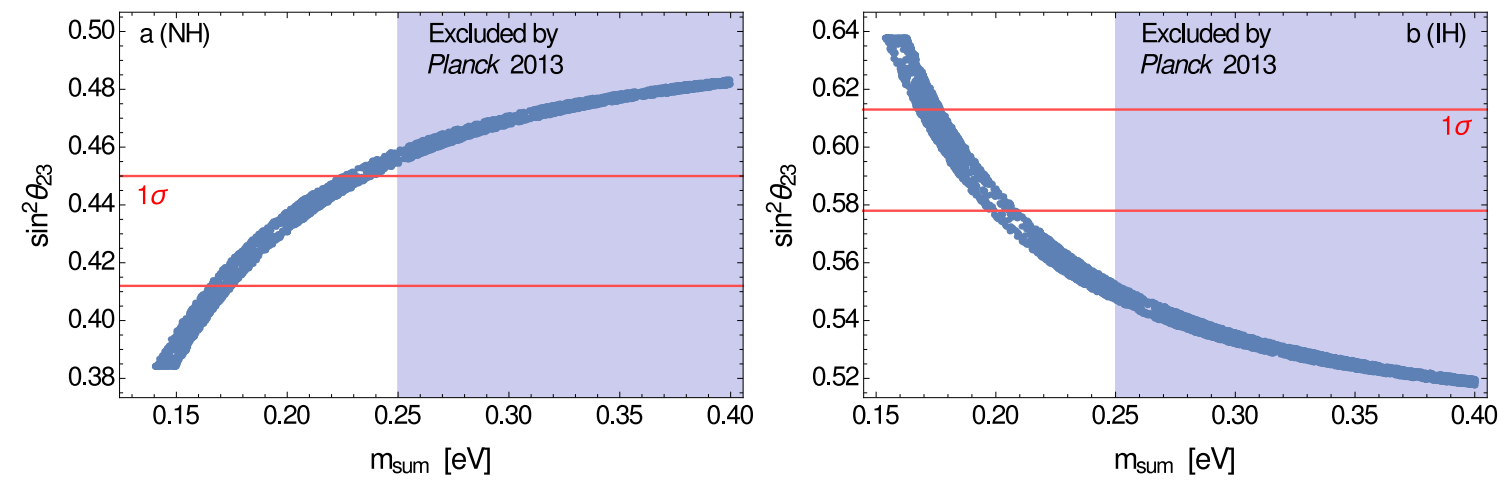

Figure 3. Scatter plots of $s_{23}^{2}$ versus $m_{\text {sum }}$ following from the constraints (3.1). Figure $3 \mathrm{a}$ is for a normal ordering of the neutrino masses and figure $3 \mathrm{~b}$ is for an inverted ordering.

dictions for $M$ (because both the moduli and phases of $M_{\mu \mu}$ and $M_{e \mu}$ are relevant). Our predictions (3.1) mean that our model features both $\left|M_{\mu \mu}\right| \ll\left|M_{\tau \tau}\right|$ and $\left|M_{e \mu}\right| \ll\left|M_{e \tau}\right|$, and this is an approximation to case $\mathrm{B}_{3}$. As a matter of fact, we have explicitly checked that the two conditions (3.1a) and (3.1b) by themselves alone lead to almost the same allowed domains as in figures $1-3$, and as in case $\mathrm{B}_{3}$ of ref. [13]. The two conditions (3.1a) and $(3.1 \mathrm{~b})$ are in practice just as predictive as that case with four predictions.

\section{The scalar potential}

\subsection{Assumptions}

In this section we investigate a way in which our class of models with three Higgs doublets and various symmetries may (i) be extended to the quark sector, and (ii) produce scalar particles with masses and couplings in agreement with the phenomenology. The aim of our investigation is to demonstrate that this can be done; we do not explore the full set of options. Thus, in this section we make additional assumptions. We stress that the validity of the models expounded in section 2 is in general independent of the specific additional assumptions that we shall utilize in this section.

Our main assumption is that there are no scalars besides the three Higgs doublets that have Yukawa couplings to the leptons. Therefore,

$$
v \equiv \sqrt{\left|v_{1}\right|^{2}+\left|v_{2}\right|^{2}+\left|v_{3}\right|^{2}}=\frac{\sqrt{2} m_{W}}{g} \approx 174 \mathrm{GeV},
$$

where $m_{W}=80.1 \mathrm{GeV}$ is the mass of the $W^{ \pm}$bosons and $g$ is the gauge-SU(2) coupling constant.

Our models have either an interchange symmetry (2.5) or the $C P$ symmetry (2.6). Those symmetries are unbroken by the Majorana mass terms of the right-handed neutrinos, which have mass dimension three. Still, those symmetries may be broken by the quadratic (i.e. mass dimension two) terms of the scalar potential. We shall assume that this does not happen, i.e. that either the interchange symmetry (2.5) or the CP symmetry (2.6) are conserved by the quadratic terms of the scalar potential. The potential is thus symmetric under 
either $\phi_{2} \leftrightarrow \phi_{3}$ or $\phi_{2} \leftrightarrow \phi_{3}^{*}$. In this paper we shall only consider the potential invariant under $\phi_{2} \leftrightarrow \phi_{3} ;{ }^{6}$ in ref. [19] the potential invariant under $\phi_{2} \leftrightarrow \phi_{3}^{*}$ has been studied.

Besides, the models have additional symmetries (2.8) together with either (2.9) or (2.10). ${ }^{7}$ The symmetry (2.8) does not involve the $\nu_{R \psi}$ and is therefore unbroken by $\mathcal{L}_{\text {Maj. }}$. We shall assume that it is also unbroken by the scalar potential; thus, the potential is invariant under $\mathbb{Z}_{2}^{(1)}: \phi_{1} \rightarrow-\phi_{1}{ }^{8}$ The symmetries $(2.9)$ or $(2.10)$, which read $\mathbb{Z}_{2}^{(2,4)}: \phi_{2} \rightarrow-\phi_{2}$ and $\mathbb{Z}_{2}^{(3,5)}: \phi_{3} \rightarrow-\phi_{3}$ in the scalar sector, are softly broken by $\mathcal{L}_{\text {Maj }}$, which is of dimension three; therefore, they must also be broken in the quadratic part of the potential. The potential therefore is

$$
\begin{aligned}
V= & \mu_{1} \phi_{1}^{\dagger} \phi_{1}+\mu_{2}\left(\phi_{2}^{\dagger} \phi_{2}+\phi_{3}^{\dagger} \phi_{3}\right)+\mu_{3}\left(\phi_{2}^{\dagger} \phi_{3}+\phi_{3}^{\dagger} \phi_{2}\right) \\
& +\lambda_{1}\left(\phi_{1}^{\dagger} \phi_{1}\right)^{2}+\lambda_{2}\left[\left(\phi_{2}^{\dagger} \phi_{2}\right)^{2}+\left(\phi_{3}^{\dagger} \phi_{3}\right)^{2}\right] \\
& +\lambda_{3} \phi_{1}^{\dagger} \phi_{1}\left(\phi_{2}^{\dagger} \phi_{2}+\phi_{3}^{\dagger} \phi_{3}\right)+\lambda_{4} \phi_{2}^{\dagger} \phi_{2} \phi_{3}^{\dagger} \phi_{3} \\
& +\lambda_{5}\left(\phi_{1}^{\dagger} \phi_{2} \phi_{2}^{\dagger} \phi_{1}+\phi_{1}^{\dagger} \phi_{3} \phi_{3}^{\dagger} \phi_{1}\right)+\lambda_{6} \phi_{2}^{\dagger} \phi_{3} \phi_{3}^{\dagger} \phi_{2} \\
& +\lambda_{7}\left[\left(\phi_{1}^{\dagger} \phi_{2}\right)^{2}+\left(\phi_{1}^{\dagger} \phi_{3}\right)^{2}\right]+\lambda_{7}^{*}\left[\left(\phi_{2}^{\dagger} \phi_{1}\right)^{2}+\left(\phi_{3}^{\dagger} \phi_{1}\right)^{2}\right] \\
& +\lambda_{8}\left[\left(\phi_{2}^{\dagger} \phi_{3}\right)^{2}+\left(\phi_{3}^{\dagger} \phi_{2}\right)^{2}\right] .
\end{aligned}
$$

The parameters $\mu_{3}$ and $\lambda_{8}$ are real because of the symmetry under $\phi_{2} \leftrightarrow \phi_{3}$. We use the freedom of rephasing $\phi_{1}$ to set $\lambda_{7}$ real too.

\subsection{The vacuum}

We assume as usual that the vacuum does not break electromagnetic invariance, i.e. that the upper components of $\phi_{1,2,3}$ have zero VEV.

The potential (4.2) may, at least for some values of its parameters, produce stability points with non-trivial relative phases among the VEVs. Those stability points are, unfortunately, hard to manipulate analytically. We shall neglect them and assume that the

\footnotetext{
${ }^{6}$ Our potential is therefore invariant under a $\mathbb{Z}_{2}$ symmetry. When that $\mathbb{Z}_{2}$ symmetry is spontaneously broken, the vacuum is two-fold degenerate. There is a minimum-energy field configuration that interpolates between the two different vacua; this is called a domain wall. The non-observation of domain walls definitely is a problem for our potential. However, we recall the reader that our analysis only purports to display a particularly simple and illustrative case; we claim our potential neither to be realistic nor to be unique. The validity of the models expounded in section 2 is independent of the specific scalar potential that we analyze in this section.

${ }^{7}$ The additional symmetries are largely arbitrary — in the construction of the models we might have chosen different additional symmetries to the same practical effect, viz. preventing each scalar doublet from having Yukawa couplings to more than one lepton doublet. Each specific additional symmmetry alters the scalar potential in a different way. Thus, in a sense the specific additional symmetries (2.8)-(2.10) constitute an assumption of this section.

${ }^{8}$ In ref. [19] a potential with quadratic terms $\phi_{1}^{\dagger} \phi_{2}, \phi_{1}^{\dagger} \phi_{3}$, and their Hermitian conjugates has been analyzed. The fit in this section does not allow for those terms, which break the symmetry $\mathbb{Z}_{2}^{(1)}$. See also footnote 6 .
} 
three VEVs $v_{k} \equiv\left\langle 0\left|\phi_{k}^{0}\right| 0\right\rangle$ are (relatively) real. The VEV of the potential is then

$$
\begin{aligned}
V_{0} \equiv\langle 0|V| 0\rangle= & \mu_{1} v_{1}^{2}+\mu_{2}\left(v_{2}^{2}+v_{3}^{2}\right)+2 \mu_{3} v_{2} v_{3} \\
& +\lambda_{1} v_{1}^{4}+\lambda_{2}\left(v_{2}^{4}+v_{3}^{4}\right)+2 l_{3} v_{1}^{2}\left(v_{2}^{2}+v_{3}^{2}\right)+2 l_{4} v_{2}^{2} v_{3}^{2},
\end{aligned}
$$

where

$$
\begin{aligned}
& l_{3} \equiv \frac{\lambda_{3}+\lambda_{5}}{2}+\lambda_{7}, \\
& l_{4} \equiv \frac{\lambda_{4}+\lambda_{6}}{2}+\lambda_{8} .
\end{aligned}
$$

The equations for vacuum stability are

$$
\begin{gathered}
0=\frac{\partial V_{0}}{\partial v_{1}^{2}}=\mu_{1}+2 \lambda_{1} v_{1}^{2}+2 l_{3}\left(v_{2}^{2}+v_{3}^{2}\right), \\
0=\frac{1}{2} \frac{\partial V_{0}}{\partial v_{2}}=\mu_{2} v_{2}+\mu_{3} v_{3}+2 \lambda_{2} v_{2}^{3}+2 l_{3} v_{1}^{2} v_{2}+2 l_{4} v_{2} v_{3}^{2}, \\
0=\frac{1}{2} \frac{\partial V_{0}}{\partial v_{3}}=\mu_{2} v_{3}+\mu_{3} v_{2}+2 \lambda_{2} v_{3}^{3}+2 l_{3} v_{1}^{2} v_{3}+2 l_{4} v_{2}^{2} v_{3} .
\end{gathered}
$$

We want a vacuum state with $v_{1} \neq 0$, because in our models one of the charged-lepton masses is proportional to $\left|v_{1}\right|$. We also want the vacuum to have $\left|v_{2}\right| \neq\left|v_{3}\right|$, because in our models $r \equiv\left|v_{2} / v_{3}\right|$ is equal to a ratio of charged-lepton masses. Fortunately, equations (4.5) have a solution with $v_{1} \neq 0$ and $v_{2} \neq \pm v_{3}$ :

$$
\begin{aligned}
& \mu_{1}=-2 \lambda_{1} v_{1}^{2}-2 l_{3}\left(v_{2}^{2}+v_{3}^{2}\right), \\
& \mu_{2}=-2 l_{3} v_{1}^{2}-2 \lambda_{2}\left(v_{2}^{2}+v_{3}^{2}\right), \\
& \mu_{3}=2\left(\lambda_{2}-l_{4}\right) v_{2} v_{3} .
\end{aligned}
$$

Plugging equations (4.6) into equation (4.3), we obtain

$$
V_{0}=\frac{\mu_{3}^{2}}{2\left(\lambda_{2}-l_{4}\right)}+\frac{\lambda_{2} \mu_{1}^{2}+\lambda_{1} \mu_{2}^{2}-2 l_{3} \mu_{1} \mu_{2}}{4\left(l_{3}^{2}-\lambda_{1} \lambda_{2}\right)}
$$

We parameterize

$$
\begin{aligned}
& v_{1}=v \sin \beta, \\
& v_{2}=-\frac{v r \cos \beta}{\sqrt{1+r^{2}}}, \\
& v_{3}=\frac{v \cos \beta}{\sqrt{1+r^{2}}},
\end{aligned}
$$

and we use $r=m_{\mu} / m_{\tau}$ (the results for either $r=m_{e} / m_{\mu}$ or $r=m_{e} / m_{\tau}$ are not qualitatively different). The angle $\beta$ will be taken to lie in the first quadrant. In this way $v_{1}$ and $v_{3}$ are positive, but this represents no lack of generality. Only the relative sign of $v_{2}$ and $v_{3}$ matters, and we have found out that the best results are obtained when $v_{2} v_{3}$ is negative. 


\subsection{The scalar mass matrices}

We expand the neutral components of the doublets as

$$
\phi_{k}^{0}=v_{k}+\frac{\rho_{k}+i \eta_{k}}{\sqrt{2}},
$$

where the fields $\rho_{k}$ and $\eta_{k}$ are real. Subsuming the terms of the potential quadratic in the fields as

$$
\begin{aligned}
V_{\text {quadratic }}= & \frac{1}{2}\left(\eta_{1}, \eta_{2}, \eta_{3}\right) M_{\eta}\left(\begin{array}{c}
\eta_{1} \\
\eta_{2} \\
\eta_{3}
\end{array}\right) \\
& +\frac{1}{2}\left(\rho_{1}, \rho_{2}, \rho_{3}\right) M_{\rho}\left(\begin{array}{c}
\rho_{1} \\
\rho_{2} \\
\rho_{3}
\end{array}\right) \\
& +\left(\phi_{1}^{-}, \phi_{2}^{-}, \phi_{3}^{-}\right) M_{\phi}\left(\begin{array}{c}
\phi_{1}^{+} \\
\phi_{2}^{+} \\
\phi_{3}^{+}
\end{array}\right),
\end{aligned}
$$

we find, by using equations (4.6), that

$$
\begin{aligned}
& M_{\eta}=4 \lambda_{7}\left(\begin{array}{ccc}
-v_{2}^{2}-v_{3}^{2} & v_{1} v_{2} & v_{1} v_{3} \\
v_{1} v_{2} & -v_{1}^{2} & 0 \\
v_{1} v_{3} & 0 & -v_{1}^{2}
\end{array}\right)+\left(2 \lambda_{2}-\lambda_{4}-\lambda_{6}+2 \lambda_{8}\right)\left(\begin{array}{ccc}
0 & 0 & 0 \\
0 & -v_{3}^{2} & v_{2} v_{3} \\
0 & v_{2} v_{3} & -v_{2}^{2}
\end{array}\right), \\
& M_{\phi}=\left(\lambda_{5}+2 \lambda_{7}\right)\left(\begin{array}{ccc}
-v_{2}^{2}-v_{3}^{2} & v_{1} v_{2} & v_{1} v_{3} \\
v_{1} v_{2} & -v_{1}^{2} & 0 \\
v_{1} v_{3} & 0 & -v_{1}^{2}
\end{array}\right)+\left(2 \lambda_{2}-\lambda_{4}\right)\left(\begin{array}{ccc}
0 & 0 & 0 \\
0 & -v_{3}^{2} & v_{2} v_{3} \\
0 & v_{2} v_{3} & -v_{2}^{2}
\end{array}\right) \\
& M_{\rho}=4\left(\begin{array}{ccc}
\lambda_{1} v_{1}^{2} & l_{3} v_{1} v_{2} & l_{3} v_{1} v_{3} \\
l_{3} v_{1} v_{2} & \lambda_{2} v_{2}^{2} & 0 \\
l_{3} v_{1} v_{3} & 0 & \lambda_{2} v_{3}^{2}
\end{array}\right)+2\left(\begin{array}{ccc}
0 & 0 & 0 \\
0 & \left(l_{4}-\lambda_{2}\right) v_{3}^{2} & \left(l_{4}+\lambda_{2}\right) v_{2} v_{3} \\
0 & \left(l_{4}+\lambda_{2}\right) v_{2} v_{3} & \left(l_{4}-\lambda_{2}\right) v_{2}^{2}
\end{array}\right)
\end{aligned}
$$

In general, the matrices $M_{\eta}$ and $M_{\phi}$ must have an eigenvector $\left(v_{1}, v_{2}, v_{3}\right)$ with eigenvalue zero, corresponding to the Goldstone bosons, hence they must be of form

$$
\begin{aligned}
M_{\eta, \phi}= & a_{\eta, \phi}\left(\begin{array}{ccc}
v_{2}^{2} & -v_{1} v_{2} & 0 \\
-v_{1} v_{2} & v_{1}^{2} & 0 \\
0 & 0 & 0
\end{array}\right)+b_{\eta, \phi}\left(\begin{array}{ccc}
v_{3}^{2} & 0 & -v_{1} v_{3} \\
0 & 0 & 0 \\
-v_{1} v_{3} & 0 & v_{1}^{2}
\end{array}\right) \\
& +c_{\eta, \phi}\left(\begin{array}{ccc}
0 & 0 & 0 \\
0 & v_{3}^{2} & -v_{2} v_{3} \\
0 & -v_{2} v_{3} & v_{2}^{2}
\end{array}\right) .
\end{aligned}
$$

In our specific case, due to the $\phi_{2} \leftrightarrow \phi_{3}$ symmetry of $V$, the coefficients $a_{\eta, \phi}=b_{\eta, \phi}$. This has the important consequence that both $M_{\eta}$ and $M_{\phi}$ are diagonalized by the orthogonal 
matrix

$$
O_{v}=\left(\begin{array}{ccc}
v_{1} / v & 0 & -v_{23} / v \\
v_{2} / v & v_{3} / v_{23} & v_{1} v_{2} /\left(v v_{23}\right) \\
v_{3} / v & -v_{2} / v_{23} & v_{1} v_{3} /\left(v v_{23}\right)
\end{array}\right),
$$

where $v_{23} \equiv \sqrt{v_{2}^{2}+v_{3}^{2}}=v \cos \beta$. We find that

$$
\begin{aligned}
& M_{\eta}^{\prime} \equiv O_{v}^{T} M_{\eta} O_{v}=\operatorname{diag}\left(0, m_{A_{2}}^{2}, m_{A_{3}}^{2}\right), \\
& M_{\phi}^{\prime} \equiv O_{v}^{T} M_{\phi} O_{v}=\operatorname{diag}\left(0, m_{\varphi_{2}}^{2}, m_{\varphi_{3}}^{2}\right),
\end{aligned}
$$

where

$$
\begin{aligned}
& m_{A_{2}}^{2}=-4 \lambda_{7} v_{1}^{2}-\left(2 \lambda_{2}-\lambda_{4}-\lambda_{6}+2 \lambda_{8}\right) v_{23}^{2}, \\
& m_{A_{3}}^{2}=-4 \lambda_{7} v^{2}, \\
& m_{\varphi_{2}}^{2}=-\left(\lambda_{5}+2 \lambda_{7}\right) v_{1}^{2}-\left(2 \lambda_{2}-\lambda_{4}\right) v_{23}^{2}, \\
& m_{\varphi_{3}}^{2}=-\left(\lambda_{5}+2 \lambda_{7}\right) v^{2} .
\end{aligned}
$$

We diagonalize $M_{\rho}$ as

$$
O_{\rho}^{T} M_{\rho} O_{\rho}=\operatorname{diag}\left(m_{H_{1}}^{2}, m_{H_{2}}^{2}, m_{H_{3}}^{2}\right)
$$

where $O_{\rho}$ is a real, orthogonal matrix. We order its columns in such a way that $m_{H_{1}}^{2} \leq$ $m_{H_{2}}^{2} \leq m_{H_{3}}^{2}$. The fields $H_{k}=\sum_{k^{\prime}=1}^{3} \rho_{k^{\prime}}\left(O_{\rho}\right)_{k^{\prime} k}$ are physical scalars with mass $m_{H_{k}}$.

\subsection{The oblique parameter $T$}

Defining

$$
F(x, y) \equiv\left\{\begin{aligned}
\frac{x+y}{2}-\frac{x y}{x-y} \ln \frac{x}{y} & \Leftarrow x \neq y, \\
0 & \Leftarrow x=y,
\end{aligned}\right.
$$

the oblique parameter $T$ is $[20]$

$$
\begin{aligned}
T= & \frac{1}{16 \pi s_{w}^{2} m_{W}^{2}}\left\{\sum_{k=2}^{3} F\left(m_{\varphi_{k}}^{2}, m_{A_{k}}^{2}\right)\right. \\
& +\sum_{k=2}^{3} \sum_{k^{\prime}=1}^{3}\left|\left(O_{v}^{T} O_{\rho}\right)_{k k^{\prime}}\right|^{2}\left[F\left(m_{\varphi_{k}}^{2}, m_{H_{k^{\prime}}}^{2}\right)-F\left(m_{A_{k}}^{2}, m_{H_{k^{\prime}}}^{2}\right)\right] \\
& +3 \sum_{k=1}^{3}\left|\left(O_{v}^{T} O_{\rho}\right)_{1 k}\right|^{2}\left[F\left(m_{Z}^{2}, m_{H_{k}}^{2}\right)-F\left(m_{W}^{2}, m_{H_{k}}^{2}\right)\right] \\
& \left.-3 F\left(m_{Z}^{2}, m_{H}^{2}\right)+3 F\left(m_{W}^{2}, m_{H}^{2}\right)\right\}
\end{aligned}
$$

where $m_{Z}$ is the $Z$-boson mass, $m_{W}$ is the $W$-boson mass, $m_{H}$ is the reference mass of the Higgs boson (which is taken to be $125 \mathrm{GeV}$ ), and $s_{w}^{2}=1-m_{W}^{2} / m_{Z}^{2}$. According to ref. [9], $-0.04<T<0.20$. 


\subsection{Extension to the quark sector}

There are many possible ways of extending our models to the quark sector. If one envisages a model with the $C P$ symmetry (2.6), then that symmetry must be broken spontaneously through $v_{2} \neq v_{3}^{*}$ and that breaking must be felt in the quark sector, because we know that there is $C P$ violation in that sector; this can be achieved only if both scalar doublets $\phi_{2}$ and $\phi_{3}$ have Yukawa couplings to the quarks. In a model with the interchange symmetry (2.5), on the other hand, $C P$ violation may proceed through complex Yukawa couplings and it is not necessary for $\phi_{2}$ and $\phi_{3}$ to couple to the quarks. Things then become much simpler because at tree level there are no flavour-changing neutral currents mediated by the neutral scalars and therefore the neutral scalars do not need to be so heavy. Thus, we extend the symmetry $\mathbb{Z}_{2}^{(1)}$ of equation (2.8) as

$$
\mathbb{Z}_{2}^{(1)}: \quad \phi_{1} \rightarrow-\phi_{1}, \quad D_{L \alpha} \rightarrow-D_{L \alpha}, \quad Q_{L k} \rightarrow-Q_{L k}, \forall k \in\{1,2,3\},
$$

where the $Q_{L k}$ are the gauge-SU(2) doublets of left-handed quarks. With this extended $\mathbb{Z}_{2}^{(1)}$, the quarks only couple to $\phi_{1}$. The Yukawa couplings of the quarks are then given by

$$
\begin{aligned}
\mathcal{L}_{\text {quark Yukawa }}= & \sum_{\chi=u, c, t} \bar{\chi} m_{\chi} \frac{-\rho_{1}+i \eta_{1} \gamma_{5}}{\sqrt{2} v_{1}} \chi-\sum_{\zeta=d, s, b} \bar{\zeta} m_{\zeta} \frac{\rho_{1}+i \eta_{1} \gamma_{5}}{\sqrt{2} v_{1}} \zeta \\
& +\left[\frac{\varphi_{1}^{+}}{v_{1}} \sum_{\chi=u, c, t} \sum_{\zeta=d, s, b} V_{\chi \zeta} \bar{\chi}\left(m_{\chi} P_{L}-m_{\zeta} P_{R}\right) \zeta+\text { H.c. }\right] \\
= & -\sum_{k=1}^{3} \frac{H_{k}\left(O_{\rho}\right)_{1 k}}{\sqrt{2} v \sin \beta}\left(\sum_{\chi=u, c, t} m_{\chi} \bar{\chi} \chi+\sum_{\zeta=d, s, b} m_{\zeta} \bar{\zeta} \zeta\right) \\
& +\frac{G^{0}-A_{3} \cot \beta}{\sqrt{2} v}\left(\sum_{\chi=u, c, t} m_{\chi} \bar{\chi} i \gamma_{5} \chi-\sum_{\zeta=d, s, b} m_{\zeta} \bar{\zeta} i \gamma_{5} \zeta\right) \\
& +\left[\frac{G^{+}-\varphi_{3}^{+} \cot \beta}{v} \sum_{\chi=u, c, t} \sum_{\zeta=d, s, b} V_{\chi \zeta} \bar{\chi}\left(m_{\chi} P_{L}-m_{\zeta} P_{R}\right) \zeta+\text { H.c. }\right],
\end{aligned}
$$

where $P_{R, L}$ are the projectors of chirality, $G^{0}$ is the neutral Goldstone boson, $G^{ \pm}$are the charged Goldstone bosons, $A_{3}$ is a physical pseudoscalar with mass $m_{A_{3}}$, and $\varphi_{3}^{ \pm}$are the physical charged scalars with mass $m_{\varphi_{3}}$. Notice in lines (4.20a) and (4.20b) the absence of flavour-changing couplings of the neutral scalars.

\subsection{Procedure for producing the scatter plots}

The input for our scatter plots is $\beta$ and the eight $\lambda_{p}(p=1, \ldots, 8)$. 
In order for the potential to be bounded from below we require that the $\lambda_{p}$ satisfy [21]

$$
\begin{aligned}
& \lambda_{1}>0, \\
& \lambda_{2}>0, \\
& L_{1}>0, \\
& L_{2}>0, \\
& L_{2} \sqrt{\lambda_{1}}+2 L_{1} \sqrt{\lambda_{2}}-4 \lambda_{2} \sqrt{\lambda_{1}}+L_{1} \sqrt{L_{2}}>0 .
\end{aligned}
$$

In inequalities (4.21),

$$
\begin{aligned}
& L_{1} \equiv 2 \sqrt{\lambda_{1} \lambda_{2}}+\lambda_{3}+\left(\lambda_{5}-2\left|\lambda_{7}\right|\right) \Theta\left(2\left|\lambda_{7}\right|-\lambda_{5}\right), \\
& L_{2} \equiv 2 \lambda_{2}+\lambda_{4}+\left(\lambda_{6}-2\left|\lambda_{8}\right|\right) \Theta\left(2\left|\lambda_{8}\right|-\lambda_{6}\right),
\end{aligned}
$$

where $\Theta$ is the step (Heaviside) function.

In order for the potential not to break unitarity we impose the following conditions on the $\lambda_{p}$, which are derived in appendix A:

$$
\begin{aligned}
& \left|\lambda_{3} \pm \lambda_{5}\right|<4 \pi, \\
& \left|\lambda_{4} \pm \lambda_{6}\right|<4 \pi, \\
& \left|\lambda_{3} \pm 2 \lambda_{7}\right|<4 \pi, \\
& \left|\lambda_{4} \pm 2 \lambda_{8}\right|<4 \pi, \\
& \left|\lambda_{3}+2 \lambda_{5} \pm 6 \lambda_{7}\right|<4 \pi, \\
& \left|\lambda_{4}+2 \lambda_{6} \pm 6 \lambda_{8}\right|<4 \pi \text {, } \\
& \left|2 \lambda_{2}-2 \lambda_{8}\right|<4 \pi, \\
& \left|2 \lambda_{2}-\lambda_{6}\right|<4 \pi, \\
& \left|6 \lambda_{2}-2 \lambda_{4}-\lambda_{6}\right|<4 \pi \text {, } \\
& \left|\lambda_{1}+\lambda_{2}+\lambda_{8} \pm \sqrt{\left(\lambda_{1}-\lambda_{2}-\lambda_{8}\right)^{2}+8 \lambda_{7}^{2}}\right|<4 \pi, \\
& \left|\lambda_{1}+\lambda_{2}+\frac{\lambda_{6}}{2} \pm \sqrt{\left(\lambda_{1}-\lambda_{2}-\frac{\lambda_{6}}{2}\right)^{2}+2 \lambda_{5}^{2}}\right|<4 \pi, \\
& \left|3 \lambda_{1}+3 \lambda_{2}+\lambda_{4}+\frac{\lambda_{6}}{2} \pm \sqrt{\left(3 \lambda_{1}-3 \lambda_{2}-\lambda_{4}-\frac{\lambda_{6}}{2}\right)^{2}+2\left(2 \lambda_{3}+\lambda_{5}\right)^{2}}\right|<4 \pi .
\end{aligned}
$$

The angle $\beta$ is an input of our scatter plots. The VEVs $v_{1,2,3}$ are determined from equations (4.8), where $v$ is given by equation (4.1) and $r=m_{\mu} / m_{\tau}$. Then, $\mu_{1}, \mu_{2}$, and $\mu_{3}$ are computed by using equations (4.6). The value of $V_{0}$ is given by equation (4.7). We require $V_{0}<0$. We also enforce a number of conditions related to the alternative stability points in appendix B:

- If the quantities in the right-hand sides of equations (B.1) are both positive, then we require $V_{0}<V_{0}^{(1 \pm)}$, where the quantities $V_{0}^{(1 \pm)}$ are given in equation (B.2). 
- If the quantity in the right-hand side of equation (B.4) is positive, then we require $V_{0}<V_{0}^{(3)}$, where $V_{0}^{(3)}$ is given in equation (B.5).

- If the quantity in the right-hand side of equation (B.8a) is positive, then we require $V_{0}<V_{0}^{(4)}$, where $V_{0}^{(4)}$ is given in equation (B.9).

- If the quantity in the right-hand side of equation (B.10) is positive (with either the plus or the minus sign), then we require $V_{0}<V_{0}^{(5 \pm)}$ (with the same sign), where $V_{0}^{(5 \pm)}$ are given in equation (B.11).

- If the quantity in the right-hand side of equation (B.12) is positive and the inequality (B.14) is satisfied, then we require $V_{0}<V_{0}^{(6)}$, with $V_{0}^{(6)}$ given in equation (B.13).

We compute the squared masses in equations (4.15). We construct $M_{\rho}$ in equation (4.11c) and diagonalize it according to equation (4.16).

We assume that the lightest physical scalar, viz. $H_{1}$, corresponds to the scalar particle discovered at $L H C$; we therefore fit its mass $m_{H_{1}}$ to be $125 \mathrm{GeV}$. This fit is very precise, hence $m_{H_{1}}$ never needs to appear in our scatter plots.

We require that the masses of the six additional scalars, i.e. $m_{\varphi_{2,3}}, m_{A_{2,3}}$, and $m_{H_{2,3}}$, are all larger than $150 \mathrm{GeV}$. We also require the parameter $T$, computed through equation (4.18), to lie in between -0.04 and +0.20 [9].

The particle discovered at LHC, which we interpret as our $H_{1}$, couples to gauge-boson pairs, to the heavy quarks, and to the $\tau$ lepton with strengths close to the predictions of the SM. We hence derive the following constraints:

- The strength of the coupling of $H_{1}$ to gauge-boson pairs, divided by the strength of the coupling of the SM Higgs boson to gauge-boson pairs, is [20] $\left|g_{Z Z}\right|{ }^{9}$ where

$$
g_{Z Z} \equiv \frac{1}{v} \sum_{k=1}^{3} v_{k}\left(O_{\rho}\right)_{k 1} .
$$

Note that $-1 \leq g_{Z Z} \leq 1$, because $g_{Z Z}$ is the scalar product of two unit vectors. The limit $\left|g_{Z Z}\right|=1$ corresponds to $H_{1}$ coupling to pairs of gauge bosons with exactly the same strength as the SM Higgs boson does. In our scatter plots we require

$$
\left|g_{Z Z}\right|>0.9 \text {. }
$$

- We observe in equation (4.20a) that $H_{1}$ couples to the quarks with strength $\left(O_{\rho}\right)_{11} / \sin \beta$ times the strength of the coupling to the quarks of the SM Higgs boson. Since the sign of $\left(O_{\rho}\right)_{11}$ is physically meaningless but is correlated with the sign of $g_{Z Z}$, we define

$$
g_{\text {quarks }} \equiv \frac{\left(O_{\rho}\right)_{11}}{\sin \beta} \frac{g_{Z Z}}{\left|g_{Z Z}\right|} .
$$

In our scatter plots we demand that $0.9<g_{\text {quarks }}<1.1$.

\footnotetext{
${ }^{9}$ The important quantity is $\left|g_{Z Z}\right|$, not $g_{Z Z}$ itself, because the sign of the first column of the matrix $O_{\rho}$ is arbitrary and physically meaningless, hence the sign of $g_{Z Z}$ is also arbitrary. Alternatively, we may reason that the physical cross sections depend on the squared amplitudes, hence on $g_{Z Z}^{2}$, not on the amplitudes themselves.
} 
- We use $\left|v_{2} / v_{3}\right|=m_{\mu} / m_{\tau}$; this means that we are assuming that, in our specific model, it is the scalar doublet $\phi_{3}$ that couples to $\overline{D_{L \tau}} \tau_{R}$. Thus, there is a Yukawa coupling

$$
\Upsilon \overline{\tau_{L}} \tau_{R}\left(v_{3}+\frac{\rho_{3}+i \eta_{3}}{\sqrt{2}}\right)+\text { H.c. }
$$

The modulus of the Yukawa coupling constant $\Upsilon$ of course is $m_{\tau} / v_{3}$. Since

$$
\rho_{3}=\sum_{k=1}^{3}\left(O_{\rho}\right)_{3 k} H_{k},
$$

$H_{1}$ couples to $\overline{\tau_{L}} \tau_{R}$ with strength $\left(O_{\rho}\right)_{31}(\Upsilon / \sqrt{2})$. The modulus of the coupling of the SM Higgs boson to $\bar{\tau}_{L} \tau_{R}$ is $m_{\tau} /(\sqrt{2} v)$. Therefore, for $H_{1}$ to couple to $\tau$ leptons with the same strength as the SM Higgs boson, one needs to have $\left|\left(O_{\rho}\right)_{31} / v_{3}\right| \approx 1 / v$. Defining

$$
g_{\tau} \equiv \frac{\left(O_{\rho}\right)_{31} v}{v_{3}} \frac{g_{Z Z}}{\left|g_{Z Z}\right|},
$$

we demand that $0.9<g_{\tau}<1.1$.

Furthermore, we see in equation (4.20c) that the physical charged scalars $\varphi_{3}^{ \pm}$interact with the quarks in the same way as the charged scalars of the type-I two-Higgs-doublet model. Therefore, in our scatter plots we have borrowed the bounds in the $\tan \beta-m_{\varphi_{3}}$ plane given in figure 18 of ref. [22].

\section{7 $\quad$ Scatter plots}

In figure 4 we plot the mass of the lightest new scalar, i.e. of $H_{2}$, against $\beta$. One sees that $\beta$ must always be close to $45^{\circ}$ and that $\beta$ becomes ever more restricted when the new-scalar masses get higher. Also notice that $m_{H_{2}}$ cannot be much higher than $300 \mathrm{GeV}$.

In figure 5 we plot $\tan \beta$ against the mass of the physical charged scalars $\varphi_{3}^{ \pm}$that interact with the quarks. Also marked in figure 5, through a solid line, is the phenomenological lower bound on the mass of $\varphi_{3}^{ \pm}$, which we have taken from figure 18 of ref. [22]. That bound incorporates the constraints from $Z \rightarrow b \bar{b}, \epsilon_{K}$, and $\Delta m_{B_{s}}$; it guarantees that the charged scalars $\varphi_{3}^{ \pm}$do not mediate excessively strong $|\Delta S|=2$ transitions through box diagrams.

In figure 6 we plot the quantities defined in equations (4.26) and (4.29) against each other. They seem to be anti-correlated; the anti-correlation becomes more well-defined when the masses of all the new scalar particles are higher.

In figure 7 we plot the eight parameters $\lambda_{p}$ of the scalar potential. One observes that $\left|\lambda_{p}\right|$ is never larger than 2 for $p \in\{1,2,7,8\}$; for $3 \leq p \leq 6$ the $\lambda_{p}$ may be somewhat larger.

In figure 8 we have plotted the quartic Higgs coupling $g_{H^{4}}$ against the cubic Higgs coupling $g_{H^{3}}$. These are the coefficients of the terms $\left(H_{1}\right)^{4}$ and $\left(H_{1}\right)^{3}$, respectively, in the Lagrangian; in the case of $g_{H^{3}}$ we have multiplied the coefficient of $\left(H_{1}\right)^{3}$ by $g_{Z Z} /\left|g_{Z Z}\right|$ in order to take into account the possibility that the field $H_{1}$ has the wrong sign. One sees that the three-Higgs coupling may be almost twenty times larger than in the SM. Also, that coupling may be zero or even negative, i.e. it may have a sign opposite to the one in the 


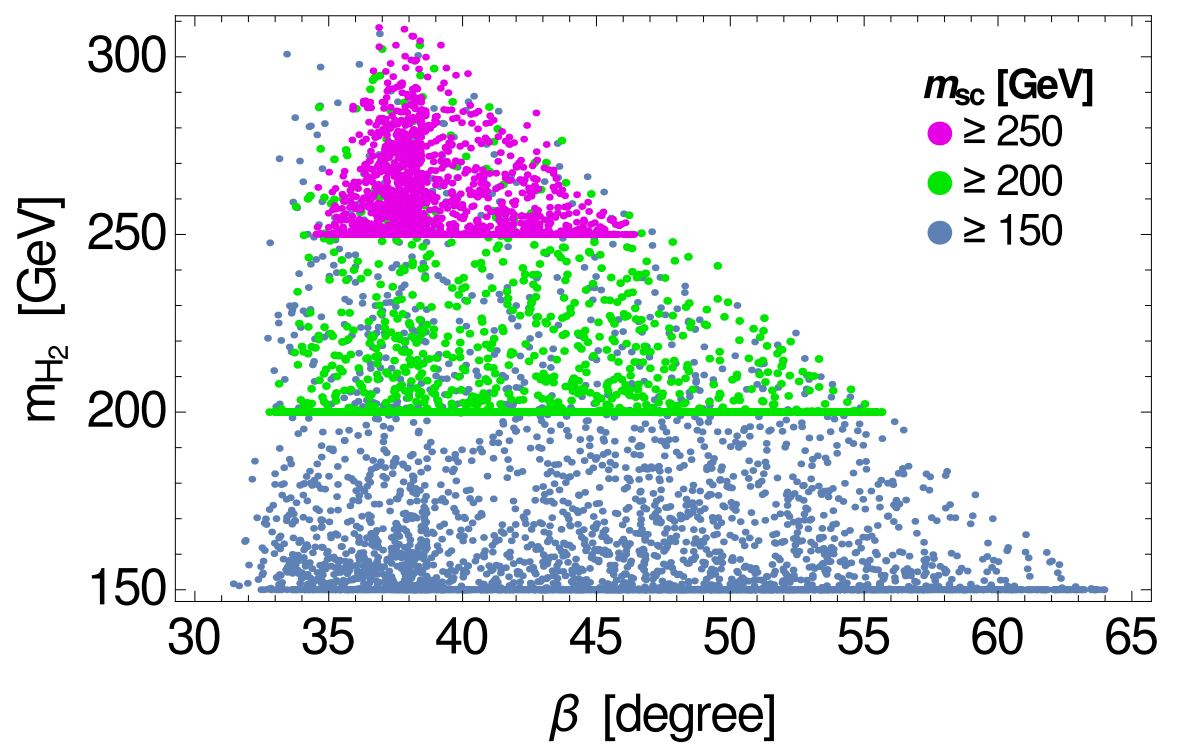

Figure 4. Scatter plot of $m_{H_{2}}$ versus the angle $\beta$. Blue points have all the scalar masses, except $m_{H_{1}}=125 \mathrm{GeV}$, higher than $150 \mathrm{GeV}$; green points have all those masses higher than $200 \mathrm{GeV}$, and magenta points have all of them higher than $250 \mathrm{GeV}$.

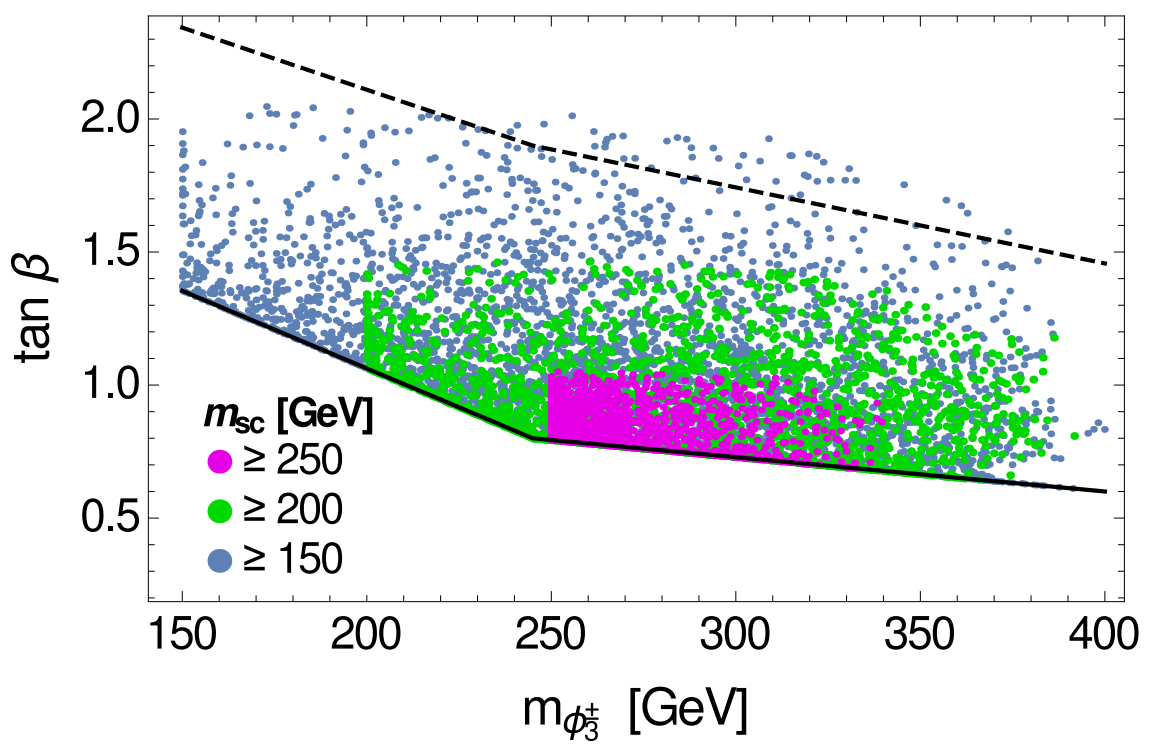

Figure 5. Scatter plot of $\tan \beta$ versus the mass of the physical charged scalars $\varphi_{3}^{ \pm}$. The notation for the colours is the same as in figure 4 . The solid line and the dashed line are phenomenological bounds extracted from figure 18 of ref. [22]. 


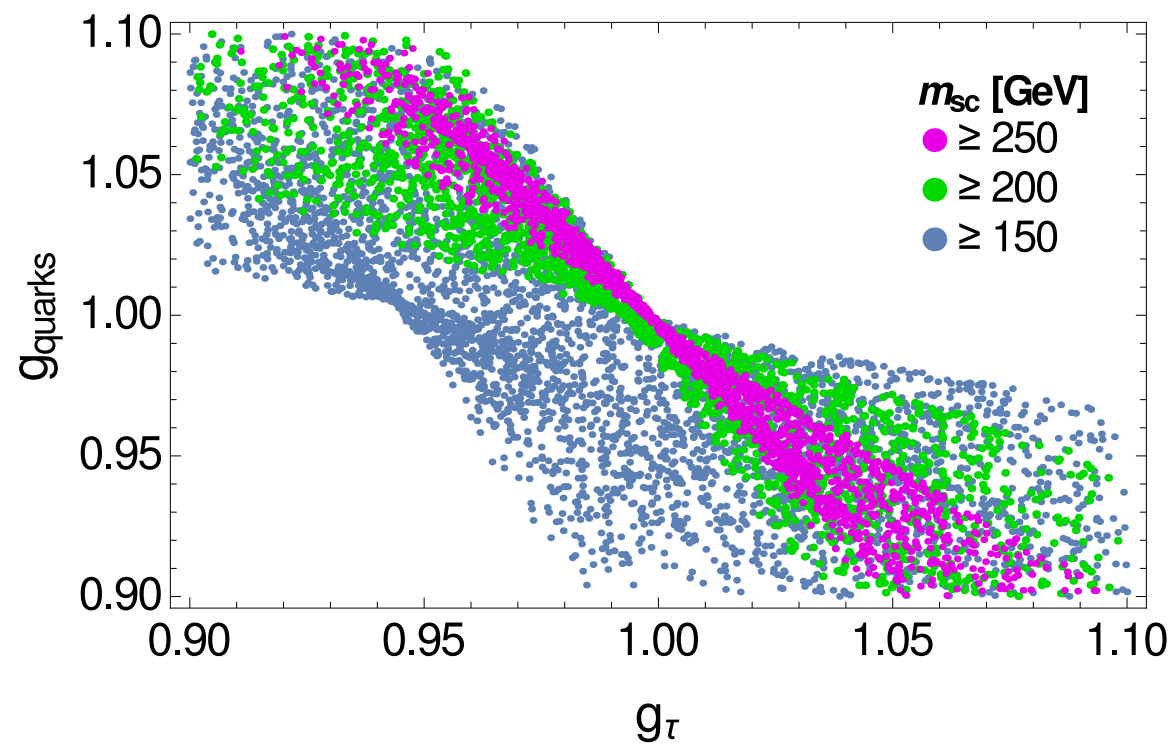

Figure 6. Scatter plot of $g_{\text {quarks }}$ versus $g_{\tau}$. The notation for the colours of the points is the same as in figure 4.

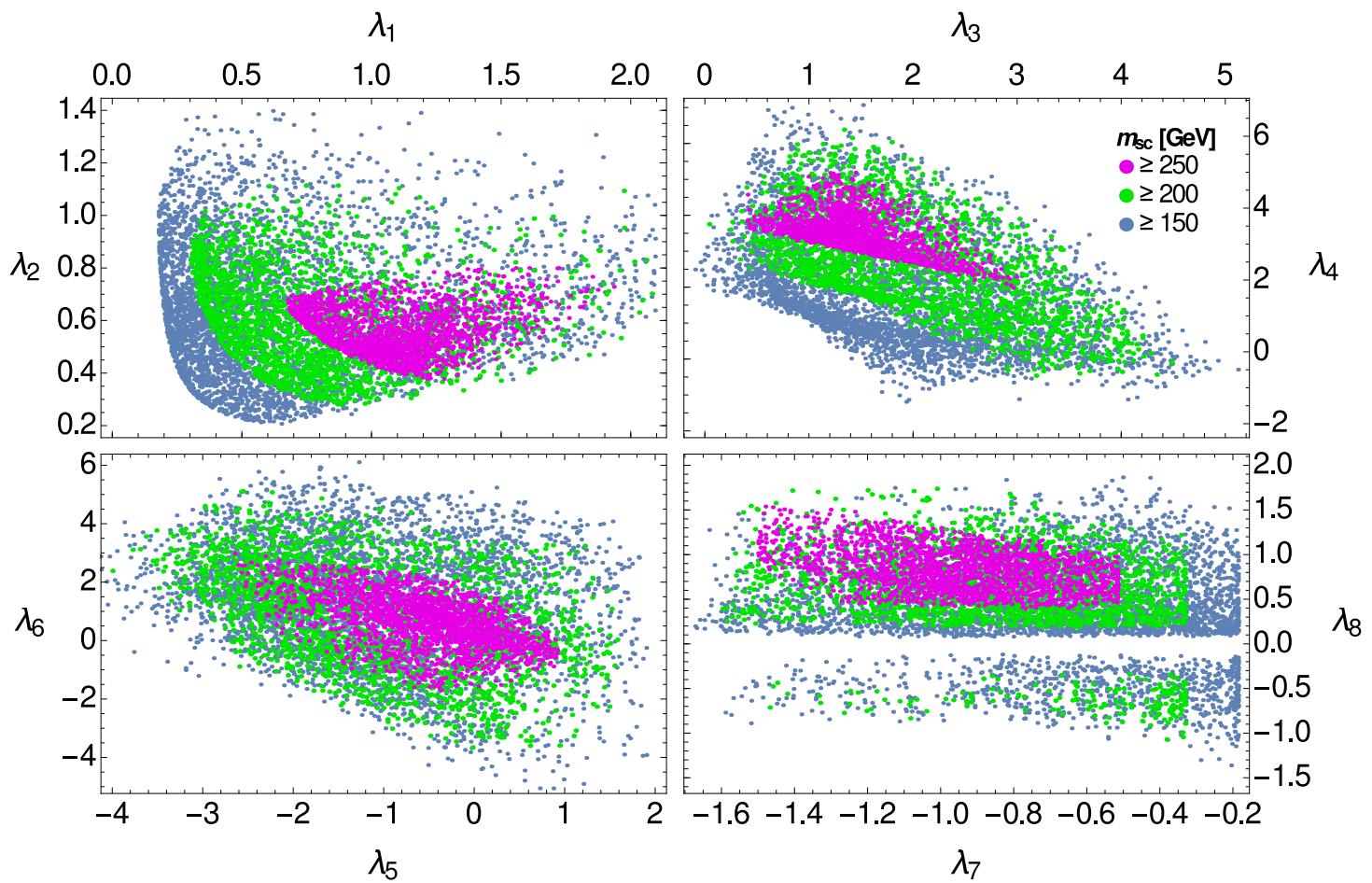

Figure 7. Scatter plots of the $\lambda_{p}$. The notation for the colours of the points is the same as in figure 4 . 


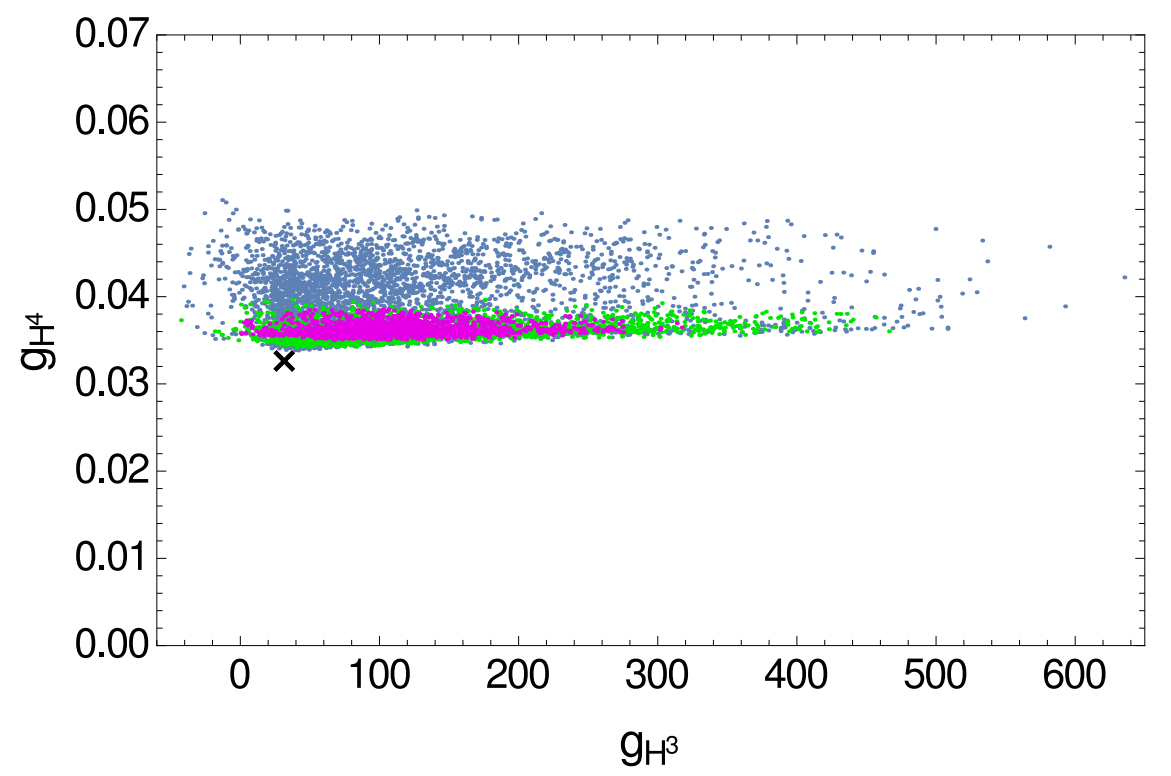

Figure 8. Scatter plot of $g_{H^{4}}$ versus $g_{H^{3}}$. The notation for the colours of the points is the same as in figure 4 . The black cross indicates the values of $g_{H^{4}}$ and $g_{H^{3}}$ in the SM.

SM. The four-Higgs coupling is always larger than the corresponding SM coupling; it may at most be $60 \%$ larger than in the SM. We point out that, in a general two-Higgs-doubletmodel, the three-Higgs coupling has less freedom (it may at most be ten times larger than in the SM) than in this model, while the four-Higgs coupling has much more freedom than in this model - it may have values from zero until almost fifteen times larger than in the $\mathrm{SM}$ [23]. Therefore, a measurement of $g_{H^{3}}$ - of the cubic interaction of the $125 \mathrm{GeV}$ scalar - may produce a large surprise and even distinguish this three-Higgs-doublet model from the most general two-Higgs-doublet one.

In figure 9 we have plotted $\left|g_{Z Z}\right|$ against the quartic Higgs coupling and against the cubic Higgs coupling. Notice that, although in our search we have restricted $\left|g_{Z Z}\right|$ to have values in the range from 0.9 to 1 , we have ended up obtaining only points with $\left|g_{Z Z}\right|>0.94$. This is because we have restricted all the scalar masses (except the one of $H_{1}$ ) to be larger than $150 \mathrm{GeV}$; larger scalar masses require a larger $\left|g_{Z Z}\right|$ because the values of $\left|g_{Z Z}\right|$ approach unity when the masses of all the new scalars are higher - this is the decoupling limit.

\section{Conclusions}

In this paper we have constructed various extensions of the SM that yield predictions for the effective light-neutrino Majorana mass matrix $M$ given in terms of the charged-lepton mass ratios. We have produced twelve models $M_{\alpha p q}$, where $\alpha \in\{e, \mu, \tau\}$ and $p, q \in\{1,2\}$. Models $M_{\alpha 1 q}$ predict

$$
\left|\frac{M_{\beta \beta}}{M_{\gamma \gamma}}\right|=\frac{m_{\beta}^{2}}{m_{\gamma}^{2}}, \quad\left|\frac{M_{\alpha \beta}}{M_{\alpha \gamma}}\right|=\frac{m_{\beta}}{m_{\gamma}}
$$



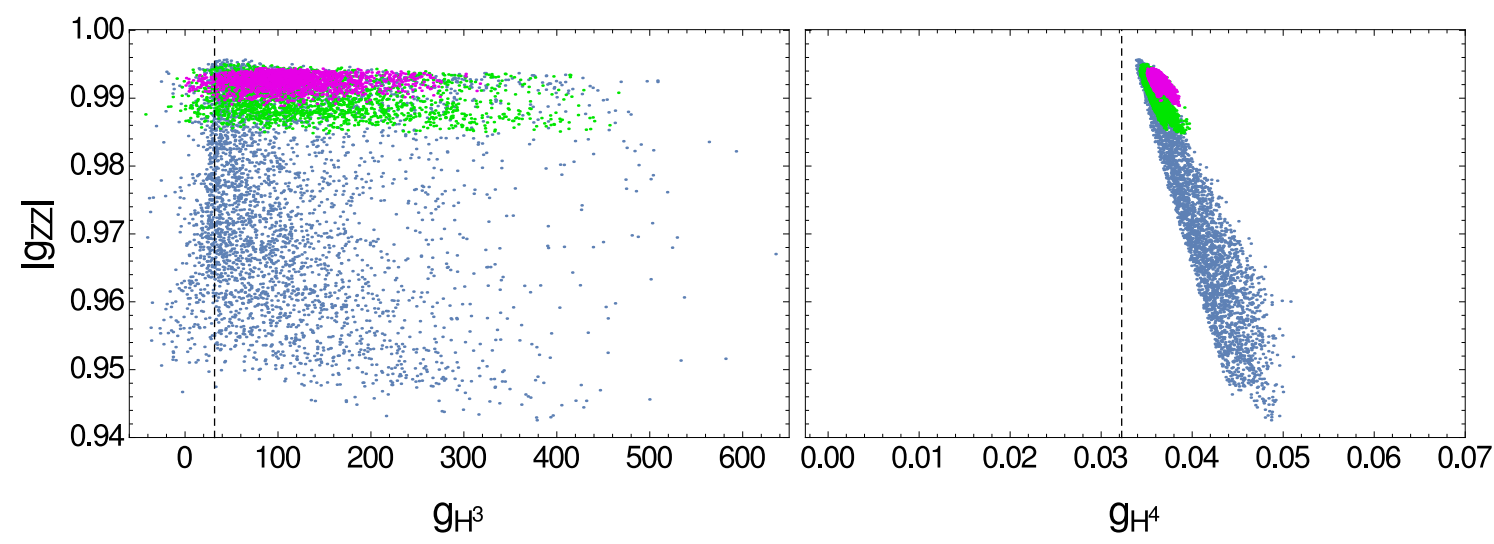

Figure 9. Scatter plots of $\left|g_{Z Z}\right|$ versus $g_{H^{3}}$ and $g_{H^{4}}$. The notation for the colours of the points is the same as in figure 4 . The dashed vertical lines indicate the values of $g_{H^{3}}$ and $g_{H^{4}}$ in the SM.

where $\alpha \neq \beta \neq \gamma \neq \alpha$, whereas models $M_{\alpha 2 q}$ predict

$$
\left|\frac{M_{\beta \beta}}{M_{\gamma \gamma}}\right|=\frac{m_{\gamma}^{2}}{m_{\beta}^{2}}, \quad\left|\frac{M_{\alpha \beta}}{M_{\alpha \gamma}}\right|=\frac{m_{\gamma}}{m_{\beta}} .
$$

Furthermore, models $M_{\alpha p 1}$ predict

$$
\arg \left[M_{\gamma \gamma}\left(M_{\alpha \beta}\right)^{2} M_{\beta \beta}^{*}\left(M_{\alpha \gamma}^{*}\right)^{2}\right]=0,
$$

whereas models $M_{\alpha p 2}$ predict

$$
\begin{aligned}
\arg \left[M_{\beta \beta}^{*} M_{\gamma \gamma}^{*}\left(M_{\beta \gamma}\right)^{2}\right] & =0, \\
\arg \left(M_{\alpha \alpha}^{*} M_{\beta \gamma}^{*} M_{\alpha \beta} M_{\alpha \gamma}\right) & =0 .
\end{aligned}
$$

In practice, the conditions (5.3) or (5.4) are not so important; this is because conditions (5.1) or (5.2) mean that two matrix elements of $M$ are relatively small and lead to our models being approximations to two-texture-zero cases. Thus, eight of our twelve models are able to correctly fit the data:

- Models $M_{\mu 1 q}$ for $q=1,2$, which are approximations to case $\mathrm{A}_{1}$.

- Models $M_{\tau 1 q}$ for $q=1,2$, which are approximations to case $\mathrm{A}_{2}$.

- Models $M_{e 1 q}$ for $q=1,2$, which are approximations to case $\mathrm{B}_{3}$.

- Models $M_{e 2 q}$ for $q=1,2$, which are approximations to case $\mathrm{B}_{4}$.

The four models $M_{\mu 2 q}$ and $M_{\tau 2 q}$ are not compatible with the phenomenological data and are therefore excluded.

We have emphasized that our models $M_{e 1 q}$ lead, just from the two conditions

$$
\left|\frac{M_{\mu \mu}}{M_{\tau \tau}}\right|=\frac{m_{\mu}^{2}}{m_{\tau}^{2}}, \quad\left|\frac{M_{e \mu}}{M_{e \tau}}\right|=\frac{m_{\mu}}{m_{\tau}}
$$


to a vast predictive power, viz. $\delta \approx 3 \pi / 2, \alpha_{21} \approx 0, \alpha_{31} \approx \pi$, and almost maximal neutrinoless double-beta decay for either a normal or an inverted neutrino mass spectrum. Moreover, the quadrant of $\theta_{23}$ is correlated with the type of mass spectrum and $\theta_{23}$ approaches $\pi / 4$ when the neutrino masses increase.

We have carefully worked out a scalar potential appropriate to our models $M_{e 1 q}$. (With slight modifications and no qualitatively different results, the potential is also appropriate to models $M_{\mu 1 q}$ and $M_{\tau 1 q}$.) Our assumptions were the following:

- There are only three Higgs doublets $\phi_{1,2,3}$.

- There is an interchange symmetry $\phi_{2} \leftrightarrow \phi_{3}$ that is not softly broken in the quadratic part of the scalar potential.

- The potential has an unbroken symmetry under $\phi_{1} \rightarrow-\phi_{1}$.

- The vacuum expectation values are real.

- The symmetry $\phi_{1} \rightarrow-\phi_{1}$ is extended to the quark sector in such a way that only $\phi_{1}$ has Yukawa couplings to the quarks; the physical neutral scalars therefore have no flavour-changing Yukawa couplings. $C P$ violation is hard, i.e. it originates in complex Yukawa couplings.

- The particle with mass $125 \mathrm{GeV}$ discovered at LHC is the lightest physical scalar.

Through a careful simulation we have found the appropriate ranges for the various parameters of the scalar potential. The physical-scalar masses cannot be much higher than a few hundred $\mathrm{GeV}$.

\section{Acknowledgments}

L.L. thanks Pedro M. Ferreira, João Paulo Silva, and Igor Ivanov for useful discussions. D.J. thanks the Lithuanian Academy of Sciences for support through the project DaFi2017. The work of L.L. is supported by the Portuguese Fundação para a Ciência e a Tecnologia through the projects CERN/FIS-NUC/0010/2015 and UID/FIS/00777/2013, which are partially funded by POCTI (FEDER), COMPETE, QREN, and the European Union.

\section{A Unitarity bounds for a 3HDM with $\mathbb{Z}_{2} \times \mathbb{Z}_{2} \times \mathbb{Z}_{2}$ symmetry}

\section{A.1 General case}

We consider the most general three-Higgs-doublet model with $\mathbb{Z}_{2}^{(1)} \times \mathbb{Z}_{2}^{(2)} \times \mathbb{Z}_{2}^{(3)}$ symmetry, where

$$
\mathbb{Z}_{2}^{(1)}: \phi_{1} \rightarrow-\phi_{1} ; \quad \mathbb{Z}_{2}^{(2)}: \phi_{2} \rightarrow-\phi_{2} ; \quad \mathbb{Z}_{2}^{(3)}: \phi_{3} \rightarrow-\phi_{3}
$$


It is immaterial in this appendix whether any of the symmetries (A.1) is softly broken; here we just deal with the quartic part of the potential

$$
\begin{aligned}
V_{\text {quartic }}= & \Lambda_{1}\left(\phi_{1}^{\dagger} \phi_{1}\right)^{2}+\Lambda_{2}\left(\phi_{2}^{\dagger} \phi_{2}\right)^{2}+\Lambda_{3}\left(\phi_{3}^{\dagger} \phi_{3}\right)^{2} \\
& +\Lambda_{4} \phi_{1}^{\dagger} \phi_{1} \phi_{2}^{\dagger} \phi_{2}+\Lambda_{5} \phi_{1}^{\dagger} \phi_{1} \phi_{3}^{\dagger} \phi_{3}+\Lambda_{6} \phi_{2}^{\dagger} \phi_{2} \phi_{3}^{\dagger} \phi_{3} \\
& +\Lambda_{7} \phi_{1}^{\dagger} \phi_{2} \phi_{2}^{\dagger} \phi_{1}+\Lambda_{8} \phi_{1}^{\dagger} \phi_{3} \phi_{3}^{\dagger} \phi_{1}+\Lambda_{9} \phi_{2}^{\dagger} \phi_{3} \phi_{3}^{\dagger} \phi_{2} \\
& +\left[\Lambda_{10}\left(\phi_{1}^{\dagger} \phi_{2}\right)^{2}+\Lambda_{11}\left(\phi_{1}^{\dagger} \phi_{3}\right)^{2}+\Lambda_{12}\left(\phi_{2}^{\dagger} \phi_{3}\right)^{2}+\text { H.c. }\right],
\end{aligned}
$$

where $\Lambda_{1, \ldots, 9}$ are real and $\Lambda_{10,11,12}$ are in general complex. We follow ref. [24] to compute the unitarity bounds on the parameters of the potential (A.2). For notational simplicity, we write

$$
\phi_{1}=\left(\begin{array}{l}
a \\
b
\end{array}\right), \quad \phi_{2}=\left(\begin{array}{l}
c \\
d
\end{array}\right), \quad \phi_{3}=\left(\begin{array}{l}
e \\
f
\end{array}\right),
$$

where the letters $a, \ldots, f$ denote creation/destruction operators as well as the corresponding particles. The (non-)existence of vacuum expectation values is immaterial for the unitarity bounds, therefore we neglect them in the notation (A.3). We denote the Hermitianconjugate operators through bars: $a^{\dagger} \rightarrow \bar{a}, b^{\dagger} \rightarrow \bar{b}$, and so on. Then,

$$
\begin{aligned}
V_{\text {quartic }}= & \Lambda_{1}(\bar{a} \bar{a} a a+\bar{b} \bar{b} b b+2 \bar{a} \bar{b} a b) \\
& +\Lambda_{2}(\bar{c} \bar{c} c c+\bar{d} \bar{d} d d+2 \bar{c} \bar{d} c d) \\
& +\Lambda_{3}(\bar{e} \bar{e} e e+\bar{f} \bar{f} f f+2 \bar{e} \bar{f} e f) \\
& +\Lambda_{4}(\bar{a} \bar{c} a c+\bar{b} \bar{d} b d+\bar{a} \bar{d} a d+\bar{b} \bar{c} b c) \\
& +\Lambda_{5}(\bar{a} \bar{e} a e+\bar{b} \bar{f} b f+\bar{a} \bar{f} a f+\bar{b} \bar{e} b e) \\
& +\Lambda_{6}(\bar{c} \bar{e} c e+\bar{d} \bar{f} d f+\bar{c} \bar{f} c f+\bar{d} \bar{e} d e) \\
& +\Lambda_{7}(\bar{a} \bar{c} a c+\bar{b} \bar{d} b d+\bar{a} \bar{d} b c+\bar{b} \bar{c} a d) \\
& +\Lambda_{8}(\bar{a} \bar{e} a e+\bar{b} \bar{f} b f+\bar{a} \bar{f} b e+\bar{b} \bar{e} a f) \\
& +\Lambda_{9}(\bar{c} \bar{e} c e+\bar{d} \bar{f} d f+\bar{c} \bar{f} d e+\bar{d} \bar{e} c f) \\
& +\Lambda_{10}(\bar{a} \bar{a} c c+\bar{b} \bar{b} d d+2 \bar{a} \bar{b} c d) \\
& +\Lambda_{10}^{*}(\bar{c} \bar{c} a a+\bar{d} \bar{d} b b+2 \bar{c} \bar{d} a b) \\
& +\Lambda_{11}(\bar{a} \bar{a} e e+\bar{b} \bar{b} f f+2 \bar{a} \bar{b} e f) \\
& +\Lambda_{11}^{*}(\bar{e} \bar{e} a a+\bar{f} \bar{f} b b+2 \bar{e} \bar{f} a b) \\
& +\Lambda_{12}(\bar{c} \bar{c} e e+\bar{d} \bar{d} f f+2 \bar{c} \bar{d} e f) \\
& +\Lambda_{12}^{*}(\bar{e} \bar{e} c c+\bar{f} \bar{f} d d+2 \bar{e} \bar{f} c d) .
\end{aligned}
$$

We must consider all the $2 \rightarrow 2$ scatterings that various pairs of particles may suffer among themselves. For instance, the three states $a a, c c$, and ee may, at tree-level, scatter through a matrix

$$
\left(\begin{array}{ccc}
2 \Lambda_{1} & 2 \Lambda_{10} & 2 \Lambda_{11} \\
2 \Lambda_{10}^{*} & 2 \Lambda_{2} & 2 \Lambda_{12} \\
2 \Lambda_{11}^{*} & 2 \Lambda_{12}^{*} & 2 \Lambda_{3}
\end{array}\right)
$$


The scattering matrices of the states $(a d, b c),(a f, b e)$, and $(b c, d e)$ are

$$
\left(\begin{array}{ll}
\Lambda_{4} & \Lambda_{7} \\
\Lambda_{7} & \Lambda_{4}
\end{array}\right), \quad\left(\begin{array}{ll}
\Lambda_{5} & \Lambda_{8} \\
\Lambda_{8} & \Lambda_{5}
\end{array}\right), \quad\left(\begin{array}{ll}
\Lambda_{6} & \Lambda_{9} \\
\Lambda_{9} & \Lambda_{6}
\end{array}\right)
$$

respectively. The scattering matrices of the states $(a \bar{d}, \bar{b} c),(a \bar{f}, \bar{b} e)$, and $(\bar{f} c, \bar{d} e)$ are

$$
\left(\begin{array}{cc}
\Lambda_{4} & 2 \Lambda_{10} \\
2 \Lambda_{10}^{*} & \Lambda_{4}
\end{array}\right), \quad\left(\begin{array}{cc}
\Lambda_{5} & 2 \Lambda_{11} \\
2 \Lambda_{11}^{*} & \Lambda_{5}
\end{array}\right), \quad\left(\begin{array}{cc}
\Lambda_{6} & 2 \Lambda_{12} \\
2 \Lambda_{12}^{*} & \Lambda_{6}
\end{array}\right)
$$

respectively. The scattering matrix of the states $(a \bar{b}, c \bar{d}, e \bar{f})$ is

$$
\left(\begin{array}{ccc}
2 \Lambda_{1} & \Lambda_{7} & \Lambda_{8} \\
\Lambda_{7} & 2 \Lambda_{2} & \Lambda_{9} \\
\Lambda_{8} & \Lambda_{9} & 2 \Lambda_{3}
\end{array}\right)
$$

The scattering matrix of the states $(\bar{a} a, \bar{b} b, \bar{c} c, \bar{d} d, \bar{e} e, \bar{f} f)$ is

$$
\left(\begin{array}{cccccc}
4 \Lambda_{1} & 2 \Lambda_{1} & \Lambda_{4}+\Lambda_{7} & \Lambda_{4} & \Lambda_{5}+\Lambda_{8} & \Lambda_{5} \\
2 \Lambda_{1} & 4 \Lambda_{1} & \Lambda_{4} & \Lambda_{4}+\Lambda_{7} & \Lambda_{5} & \Lambda_{5}+\Lambda_{8} \\
\Lambda_{4}+\Lambda_{7} & \Lambda_{4} & 4 \Lambda_{2} & 2 \Lambda_{2} & \Lambda_{6}+\Lambda_{9} & \Lambda_{6} \\
\Lambda_{4} & \Lambda_{4}+\Lambda_{7} & 2 \Lambda_{2} & 4 \Lambda_{2} & \Lambda_{6} & \Lambda_{6}+\Lambda_{9} \\
\Lambda_{5}+\Lambda_{8} & \Lambda_{5} & \Lambda_{6}+\Lambda_{9} & \Lambda_{6} & 4 \Lambda_{3} & 2 \Lambda_{3} \\
\Lambda_{5} & \Lambda_{5}+\Lambda_{8} & \Lambda_{6} & \Lambda_{6}+\Lambda_{9} & 2 \Lambda_{3} & 4 \Lambda_{3}
\end{array}\right)
$$

In order to guarantee unitarity, we must enforce the condition that the moduli of all the eigenvalues of these matrices (and of a few more analogous matrices) are smaller than $4 \pi$. After some effort we find that those eigenvalues are

$$
\begin{array}{lll}
\Lambda_{4} \pm \Lambda_{7}, & \Lambda_{5} \pm \Lambda_{8}, & \Lambda_{6} \pm \Lambda_{9} \\
\Lambda_{4} \pm 2\left|\Lambda_{10}\right|, & \Lambda_{5} \pm 2\left|\Lambda_{11}\right|, & \Lambda_{6} \pm 2\left|\Lambda_{12}\right|, \\
\Lambda_{4}+2 \Lambda_{7} \pm 6\left|\Lambda_{10}\right|, & \Lambda_{5}+2 \Lambda_{8} \pm 6\left|\Lambda_{11}\right|, & \Lambda_{6}+2 \Lambda_{9} \pm 6\left|\Lambda_{12}\right|
\end{array}
$$

and the eigenvalues of the matrices (A.5), (A.8), and

$$
\left(\begin{array}{ccc}
6 \Lambda_{1} & 2 \Lambda_{4}+\Lambda_{7} & 2 \Lambda_{5}+\Lambda_{8} \\
2 \Lambda_{4}+\Lambda_{7} & 6 \Lambda_{2} & 2 \Lambda_{6}+\Lambda_{9} \\
2 \Lambda_{5}+\Lambda_{8} & 2 \Lambda_{6}+\Lambda_{9} & 6 \Lambda_{3}
\end{array}\right)
$$




\section{A.2 Case with additional symmetry $\phi_{2} \leftrightarrow \phi_{3}$}

In our case there is an additional symmetry $\phi_{2} \leftrightarrow \phi_{3}$ in the potential, and that simplifies things much. Comparing equations (4.2) and (A.2), we see that

$$
\begin{aligned}
\Lambda_{1} & \rightarrow \lambda_{1}, \\
\Lambda_{2}, \Lambda_{3} & \rightarrow \lambda_{2}, \\
\Lambda_{4}, \Lambda_{5} & \rightarrow \lambda_{3}, \\
\Lambda_{6} & \rightarrow \lambda_{4}, \\
\Lambda_{7}, \Lambda_{8} & \rightarrow \lambda_{5}, \\
\Lambda_{9} & \rightarrow \lambda_{6}, \\
\Lambda_{10}, \Lambda_{11} & \rightarrow \lambda_{7}, \\
\Lambda_{12} & \rightarrow \lambda_{8} .
\end{aligned}
$$

The quantities (A.10) then become

$$
\begin{array}{ll}
\lambda_{3} \pm \lambda_{5}, & \lambda_{4} \pm \lambda_{6}, \\
\lambda_{3} \pm 2 \lambda_{7}, & \lambda_{4} \pm 2 \lambda_{8}, \\
\lambda_{3}+2 \lambda_{5} \pm 6 \lambda_{7}, & \lambda_{4}+2 \lambda_{6} \pm 6 \lambda_{8},
\end{array}
$$

and the matrices (A.5), (A.8), and (A.11) become

$$
\begin{gathered}
\left(\begin{array}{ccc}
2 \lambda_{1} & 2 \lambda_{7} & 2 \lambda_{7} \\
2 \Lambda_{7} & 2 \lambda_{2} & 2 \lambda_{8} \\
2 \lambda_{7} & 2 \lambda_{8} & 2 \lambda_{2}
\end{array}\right), \quad\left(\begin{array}{ccc}
2 \lambda_{1} & \lambda_{5} & \lambda_{5} \\
\lambda_{5} & 2 \lambda_{2} & \lambda_{6} \\
\lambda_{5} & \lambda_{6} & 2 \lambda_{2}
\end{array}\right), \\
\left(\begin{array}{ccc}
6 \lambda_{1} & 2 \lambda_{3}+\lambda_{5} & 2 \lambda_{3}+\lambda_{5} \\
2 \lambda_{3}+\lambda_{8} & 6 \lambda_{2} & 2 \lambda_{4}+\lambda_{6} \\
2 \lambda_{3}+\lambda_{5} & 2 \lambda_{4}+\lambda_{6} & 6 \lambda_{2}
\end{array}\right) .
\end{gathered}
$$

The matrices (A.14) are 2-3 symmetric and therefore their eigenvalues are easy to find. One thus obtains the quantities in the left-hand sides of inequalities (4.23).

\section{B Other stability points}

Besides the vacuum state given by equations (4.6) and (4.7), there are several other stability points of the potential. The vacuum state must have a lower value of the potential than all other stability points. Therefore we must consider as many stability points as we can and, for each of them, compute the expectation value of the potential. That is what we do in the following.

1. Equations (4.5) have solutions with $v_{1} \neq 0$ and $v_{3}= \pm v_{2}$. They are

$$
\begin{aligned}
& v_{1}^{2}=\frac{2\left(\lambda_{2}+l_{4}\right) \mu_{1}-4 l_{3}\left(\mu_{2} \pm \mu_{3}\right)}{8 l_{3}^{2}-4 \lambda_{1}\left(\lambda_{2}+l_{4}\right)}, \\
& v_{2}^{2}=\frac{-2 l_{3} \mu_{1}+2 \lambda_{1}\left(\mu_{2} \pm \mu_{3}\right)}{8 l_{3}^{2}-4 \lambda_{1}\left(\lambda_{2}+l_{4}\right)} .
\end{aligned}
$$


Plugging $v_{3}= \pm v_{2}$ together with equations (B.1) into equation (4.3), one obtains

$$
V_{0}=V_{0}^{(1 \pm)} \equiv \frac{\left(\lambda_{2}+l_{4}\right) \mu_{1}^{2}+2 \lambda_{1}\left(\mu_{2} \pm \mu_{3}\right)^{2}-4 l_{3} \mu_{1}\left(\mu_{2} \pm \mu_{3}\right)}{8 l_{3}^{2}-4 \lambda_{1}\left(\lambda_{2}+l_{4}\right)} .
$$

2. The point $v_{1}=v_{2}=v_{3}=0$ has

$$
V_{0}=V_{0}^{(2)} \equiv 0
$$

3. If $v_{2}=v_{3}=0$ but $v_{1} \neq 0$, there is a stability point with

$$
v_{1}^{2}=-\frac{\mu_{1}}{2 \lambda_{1}},
$$

yielding

$$
V_{0}=V_{0}^{(3)} \equiv-\frac{\mu_{1}^{2}}{4 \lambda_{1}}
$$

4. If $v_{1}=0$ but $v_{2} \neq 0$ and $v_{3} \neq 0$, we may analytically entertain the possibility that the VEVs of $\phi_{2}^{0}$ and $\phi_{3}^{0}$ have a relative phase $\vartheta$. We take in this case both $v_{2}$ and $v_{3}$ to be positive and

$$
\begin{aligned}
V_{0}= & \mu_{2}\left(v_{2}^{2}+v_{3}^{2}\right)+\lambda_{2}\left(v_{2}^{4}+v_{3}^{4}\right)+\left(\lambda_{4}+\lambda_{6}\right) v_{2}^{2} v_{3}^{2} \\
& +2 \mu_{3} v_{2} v_{3} \cos \vartheta+2 \lambda_{8} v_{2}^{2} v_{3}^{2} \cos (2 \vartheta) .
\end{aligned}
$$

The stationarity equations are

$$
\begin{aligned}
& 0=\mu_{3} \sin \vartheta+2 \lambda_{8} v_{2} v_{3} \sin (2 \vartheta), \\
& 0=\mu_{2} v_{2}+\mu_{3} v_{3} \cos \vartheta+2 \lambda_{2} v_{2}^{3}+\left(\lambda_{4}+\lambda_{6}\right) v_{2} v_{3}^{2}+2 \lambda_{8} v_{2} v_{3}^{2} \cos (2 \vartheta), \\
& 0=\mu_{2} v_{3}+\mu_{3} v_{2} \cos \vartheta+2 \lambda_{2} v_{3}^{3}+\left(\lambda_{4}+\lambda_{6}\right) v_{2}^{2} v_{3}+2 \lambda_{8} v_{2}^{2} v_{3} \cos (2 \vartheta) .
\end{aligned}
$$

This leads to the following possibilities:

(a) $\cos \vartheta= \pm 1$ and $v_{3} \neq v_{2}$. Then,

$$
\begin{aligned}
v_{2}^{2}+v_{3}^{2} & =-\frac{\mu_{2}}{2 \lambda_{2}}, \\
\left(2 \lambda_{2}-\lambda_{4}-\lambda_{6}-2 \lambda_{8}\right) v_{2} v_{3} & = \pm \mu_{3} .
\end{aligned}
$$

Plugging $\cos \vartheta= \pm 1$ and equations (B.8) into equation (B.6), one obtains

$$
V_{0}=V_{0}^{(4)} \equiv-\frac{\mu_{2}^{2}}{4 \lambda_{2}}+\frac{\mu_{3}^{2}}{2 \lambda_{2}-\lambda_{4}-\lambda_{6}-2 \lambda_{8}} .
$$

(b) $\cos \vartheta= \pm 1$ and $v_{3}=v_{2}$. One then has

$$
v_{2}^{2}=-\frac{\mu_{2} \pm \mu_{3}}{2 \lambda_{2}+\lambda_{4}+\lambda_{6}+2 \lambda_{8}},
$$

leading to

$$
V_{0}=V_{0}^{(5 \pm)} \equiv-\frac{\left(\mu_{2} \pm \mu_{3}\right)^{2}}{2 \lambda_{2}+\lambda_{4}+\lambda_{6}+2 \lambda_{8}}
$$


(c) $\cos \vartheta=-\mu_{3} /\left(4 \lambda_{8} v_{2} v_{3}\right)$. This leads to

$$
v_{3}^{2}=v_{2}^{2}=-\frac{\mu_{2}}{2 \lambda_{2}+\lambda_{4}+\lambda_{6}-2 \lambda_{8}}
$$

and to

$$
V_{0}=V_{0}^{(6)} \equiv-\frac{\mu_{2}^{2}}{2 \lambda_{2}+\lambda_{4}+\lambda_{6}-2 \lambda_{8}}-\frac{\mu_{3}^{2}}{4 \lambda_{8}} .
$$

Of course, this stability point only exists if $|\cos \vartheta| \leq 1$, viz.

$$
1 \leq\left|\frac{4 \lambda_{8} \mu_{2}}{\left(2 \lambda_{2}+\lambda_{4}+\lambda_{6}-2 \lambda_{8}\right) \mu_{3}}\right| .
$$

Open Access. This article is distributed under the terms of the Creative Commons Attribution License (CC-BY 4.0), which permits any use, distribution and reproduction in any medium, provided the original author(s) and source are credited.

\section{References}

[1] P. Minkowski, $\mu \rightarrow$ er at a Rate of One Out of $10^{9}$ Muon Decays?, Phys. Lett. B 67 (1977) 421 [inSPIRE].

[2] T. Yanagida, Horizontal gauge symmetry and masses of neutrinos, in Proceedings of the workshop on unified theory and baryon number in the universe, Tsukuba, Japan (1979), O. Sawata and A. Sugamoto eds., KEK report 79-18, Tsukuba (1979) [INSPIRE].

[3] S.L. Glashow, The future of elementary particle physics, in Quarks and leptons, proceedings of the advanced study institute, Cargèse, Corsica, (1979), M. Lévy et al. eds., Plenum, New York (1980) [INSPIRE].

[4] M. Gell-Mann, P. Ramond and R. Slansky, Complex spinors and unified theories, in Supergravity, D.Z. Freedman and F. van Nieuwenhuizen eds., North Holland, Amsterdam (1979) [INSPIRE].

[5] R.N. Mohapatra and G. Senjanović, Neutrino Mass and Spontaneous Parity Violation, Phys. Rev. Lett. 44 (1980) 912 [INSPIRE].

[6] W. Grimus and L. Lavoura, Softly broken lepton numbers and maximal neutrino mixing, JHEP 07 (2001) 045 [hep-ph/0105212] [INSPIRE].

[7] W. Grimus and L. Lavoura, Softly broken lepton numbers: An Approach to maximal neutrino mixing, Acta Phys. Polon. B 32 (2001) 3719 [hep-ph/0110041] [INSPIRE].

[8] W. Grimus and L. Lavoura, Leptogenesis in seesaw models with a twofold degenerate neutrino Dirac mass matrix, J. Phys. G 30 (2004) 1073 [hep-ph/0311362] [INSPIRE].

[9] Particle Data Group collaboration, C. Patrignani et al., Review of Particle Physics, Chin. Phys. C 40 (2016) 100001 [InSPIRE].

[10] S.F. King, Unified Models of Neutrinos, Flavour and CP-violation, Prog. Part. Nucl. Phys. 94 (2017) 217 [arXiv:1701.04413] [INSPIRE].

[11] S.F. King, A. Merle, S. Morisi, Y. Shimizu and M. Tanimoto, Neutrino Mass and Mixing: from Theory to Experiment, New J. Phys. 16 (2014) 045018 [arXiv:1402.4271] [InSPIRE].

[12] G. Altarelli and F. Feruglio, Discrete Flavor Symmetries and Models of Neutrino Mixing, Rev. Mod. Phys. 82 (2010) 2701 [arXiv:1002.0211] [INSPIRE]. 
[13] P.H. Frampton, S.L. Glashow and D. Marfatia, Zeroes of the neutrino mass matrix, Phys. Lett. B 536 (2002) 79 [hep-ph/0201008] [INSPIRE].

[14] P.F. de Salas, D.V. Forero, C.A. Ternes, M. Tórtola and J.W.F. Valle, Status of neutrino oscillations 2017, arXiv:1708.01186 [INSPIRE].

[15] Planck collaboration, P.A.R. Ade et al., Planck 2013 results. XVI. Cosmological parameters, Astron. Astrophys. 571 (2014) A16 [arXiv: 1303.5076] [INSPIRE].

[16] R. Emami et al., Evidence of Neutrino Enhanced Clustering in a Complete Sample of Sloan Survey Clusters, Implying $\sum m_{\nu}=0.11 \pm 0.03 \mathrm{eV}$, arXiv:1711.05210 [INSPIRE].

[17] J.M. Lamprea and E. Peinado, Seesaw scale discrete dark matter and two-zero texture Majorana neutrino mass matrices, Phys. Rev. D 94 (2016) 055007 [arXiv:1603.02190] [INSPIRE].

[18] W. Grimus, A.S. Joshipura, L. Lavoura and M. Tanimoto, Symmetry realization of texture zeros, Eur. Phys. J. C 36 (2004) 227 [hep-ph/0405016] [INSPIRE].

[19] P.M. Ferreira, W. Grimus, D. Jurciukonis and L. Lavoura, Scotogenic model for co-bimaximal mixing, JHEP 07 (2016) 010 [arXiv: 1604.07777] [INSPIRE].

[20] W. Grimus, L. Lavoura, O.M. Ogreid and P. Osland, A Precision constraint on multi-Higgs-doublet models, J. Phys. G 35 (2008) 075001 [arXiv:0711.4022] [INSPIRE].

[21] K. Kannike, Vacuum Stability Conditions From Copositivity Criteria, Eur. Phys. J. C 72 (2012) 2093 [arXiv: 1205.3781] [InSPIRE].

[22] G.C. Branco, P.M. Ferreira, L. Lavoura, M.N. Rebelo, M. Sher and J.P. Silva, Theory and phenomenology of two-Higgs-doublet models, Phys. Rept. 516 (2012) 1 [arXiv:1106.0034] [INSPIRE].

[23] L. Lavoura and D. Jurčiukonis, Unitarity bounds and the allowed Higgs masses and couplings in a general $2 H D M$, in preparation.

[24] M.P. Bento, H.E. Haber, J.C. Romão and J.P. Silva, Multi-Higgs doublet models: physical parametrization, sum rules and unitarity bounds, JHEP 11 (2017) 095 [arXiv:1708.09408] [INSPIRE]. 